\title{
On the structure of signed Selmer groups
}

\section{Gautier Ponsinet ${ }^{1}$}

Received: 19 July 2018 / Accepted: 22 April 2019 / Published online: 17 May 2019

(c) The Author(s) 2019

\begin{abstract}
Let $F$ be a number field unramified at an odd prime $p$ and $F_{\infty}$ be the $\mathbf{Z}_{p}$-cyclotomic extension of $F$. Generalizing Kobayashi plus/minus Selmer groups for elliptic curves, Büyükboduk and Lei have defined modified Selmer groups, called signed Selmer groups, for certain non-ordinary $\operatorname{Gal}(\bar{F} / F)$-representations. In particular, their construction applies to abelian varieties defined over $F$ with good supersingular reduction at primes of $F$ dividing $p$. Assuming that these Selmer groups are cotorsion $\mathbf{Z}_{p}\left[\left[\mathrm{Gal}\left(F_{\infty} / F\right)\right]\right]$-modules, we show that they have no proper sub- $\mathbf{Z}_{p}\left[\left[\mathrm{Gal}\left(F_{\infty} / F\right)\right]\right]$-module of finite index. We deduce from this a number of arithmetic applications. On studying the Euler-Poincaré characteristic of these Selmer groups, we obtain an explicit formula on the size of the Bloch-Kato Selmer group attached to these representations. Furthermore, for two such representations that are isomorphic modulo $p$, we compare the Iwasawa-invariants of their signed Selmer groups.
\end{abstract}

Keywords Iwasawa theory $\cdot$ Supersingular primes $\cdot$ Abelian varieties

Mathematics Subject Classification 11R23 (Primary) $\cdot 11 \mathrm{G} 10 \cdot 11 \mathrm{R} 18$

\section{Contents}

Introduction . . . . . . . . . . . . . . . . . . . . . . . . . 1636

1 Coleman maps and signed Selmer groups . . . . . . . . . . . . . . . . . . . . . . . . 1637

1.1 Cyclotomic extension and Iwasawa algebra . . . . . . . . . . . . . . . . . . . . . . . . . . . . . . . . . . . . . . . . . . . . . . . . . . .

1.2 Motives . . . . . . . . . . . . . . . . . . . . . . . . . 1638

1.3 Dieudonné modules . . . . . . . . . . . . . . . . . . . . . . . . . . . . . 1639

1.4 Decomposition of Perrin-Riou's big logarithm map . . . . . . . . . . . . . . . . . . . . . . 1639

1.5 Signed Coleman maps . . . . . . . . . . . . . . . . . . . . . . . . . . . . . . . . . . . . 1640

1.6 Signed Selmer groups . . . . . . . . . . . . . . . . . . . . . . . . . . . . . . . . . . . . . . . . . . . . . . . . . . . . . . . . . . . . . .

1.7 Bloch-Kato's Selmer groups . . . . . . . . . . . . . . . . . . . . . . . . . . . . . . . . . . . . . . . . . . . . . . . . . . . . . . . . . .

2 Submodules of finite index . . . . . . . . . . . . . . . . . . . . . . . . . . . . 1644

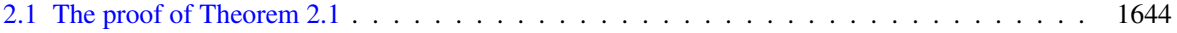

2.2 An application: computation of the Euler-Poincaré characteristic . . . . . . . . . . . . . . 1649

3 Congruences . . . . . . . . . . . . . . . . . . . . . . . 1650

3.1 Wach modules . . . . . . . . . . . . . . . . . . . . . . . . 1651

3.2 Congruences of signed Coleman maps . . . . . . . . . . . . . . . . . . . . . . 1651

Gautier Ponsinet

gautier.ponsinet@mpim-bonn.mpg.de

1 Max Planck Institut for Mathematics, Vivatsgasse 7, 53111 Bonn, Germany 
3.3 Non-primitive signed Selmer groups . . . . . . . . . . . . . . . . . . . . . . . . . . 1653

References . . . . . . . . . . . . . . . . . . . . . . . 1657

\section{Introduction}

Let $F$ be a number field and $E$ be an elliptic curve defined over $F$. Let $p$ be an odd prime and $F_{\infty}$ the $\mathbf{Z}_{p}$-cyclotomic extension of $F$ (see Sect. 1.1). On the algebraic side of the Iwasawa theory for $E$ developed by Mazur [22] is the $p$-Selmer group associated to $E$ over $F_{\infty}$, denoted by $\operatorname{Sel}_{p}\left(E / F_{\infty}\right)$, which is naturally a discrete $\mathbf{Z}_{p}\left[\left[\mathrm{Gal}\left(F_{\infty} / F\right)\right]\right]$-module. The Selmer group contains arithmetic information of the curve, e.g. it fits in the exact sequence of groups

$$
0 \rightarrow E\left(F_{\infty}\right) \otimes \mathbf{Q}_{p} / \mathbf{Z}_{p} \rightarrow \operatorname{Sel}_{p}\left(E / F_{\infty}\right) \rightarrow \amalg_{p}\left(E / F_{\infty}\right) \rightarrow 0,
$$

where $E\left(F_{\infty}\right)$ is the group of $F_{\infty}$-rational points and $\amalg_{p}\left(E / F_{\infty}\right)$ the $p$-primary component of the Tate-Shafarevich group of $E$ over $F_{\infty}$. One goal of Iwasawa theory is to understand the structure of $\operatorname{Sel}_{p}\left(E / F_{\infty}\right)$ as $\mathbf{Z}_{p}\left[\left[\operatorname{Gal}\left(F_{\infty} / F\right)\right]\right]$-module.

When $E$ has good ordinary reduction at primes of $F$ dividing $p$, a conjecture of Mazur (proved by Kato [14] when $F=\mathbf{Q}$ ) states that the Pontryagin dual of $\operatorname{Sel}_{p}\left(E / F_{\infty}\right)$ is a torsion $\mathbf{Z}_{p}\left[\left[\mathrm{Gal}\left(F_{\infty} / F\right)\right]\right]$-module. Furthemore, assuming Mazur's conjecture and that the $E(F)$ has no $p$-torsion, Greenberg [10, Proposition 4.14] showed that $\operatorname{Sel}_{p}\left(E / F_{\infty}\right)$ has no proper sub- $\mathbf{Z}_{p}\left[\left[\mathrm{Gal}\left(F_{\infty} / F\right)\right]\right]$-module of finite index.

When $E$ has good supersingular reduction at some prime above $p$, the Selmer group over $F_{\infty}$ is no longer a cotorsion $\mathbf{Z}_{p}\left[\left[\operatorname{Gal}\left(F_{\infty} / F\right)\right]\right]$-module. In the case $F=\mathbf{Q}$ and $a_{p}=0$ (holds whenever $p \geqslant 5$ ), Kobayashi [19] has defined the so called plus and minus (or signed) Selmer groups, and proves that the Pontryagin duals of these Selmer groups are torsion $\mathbf{Z}_{p}\left[\left[\operatorname{Gal}\left(\mathbf{Q}_{\infty} / \mathbf{Q}\right)\right]\right]$-modules. Kim [16] has then extended the definition of these Selmer groups to number fields $F$ where $p$ is unramified and generalized Greenberg's result and showed that if the signed Selmer groups of $E$ over $F_{\infty}$ are cotorsion $\mathbf{Z}_{p}\left[\left[\operatorname{Gal}\left(F_{\infty} / F\right)\right]\right]$ modules, then they have no proper submodule of finite index (for one of the signed Selmer group, namely the plus one, he requires the additional assumption that $p$ splits completely in $F$ and is totally ramified in $F_{\infty}$ ). This assumption has recently been removed by Kitajima and Otsuki, see [18].

Kobayashi's construction of signed Selmer groups has been generalized to many situations $[5,12,17,20]$. In [5], using $p$-adic Hodge theory machinery, Büyükboduk and Lei have defined signed Selmer groups for certain non-ordinary Galois representations of $\operatorname{Gal}(\bar{F} / F)$ (see, Sects. 1.2 and 1.3 for hypotheses). In particular, their construction applies to abelian varieties defined over $F$ with good supersingular reduction at primes of $F$ dividing $p$. The definition of the signed Selmer groups depends on a choice of a basis for the Dieudonné module associated to the representation. For such a basis, we may attach to each of its subset $\underline{I}$ of some prescribed cardinality a signed Selmer group, which we denote in this introduction by $\operatorname{Sel}_{\underline{I}}\left(T / F_{\infty}\right.$ ), where $T$ is a Galois representation (a free $\mathbf{Z}_{p}$-module of finite rank with a continuous action of the absolute Galois group of $F$ ) to which the construction of op cit. applies. They conjectured these signed Selmer groups to be cotorsion $\mathbf{Z}_{p}\left[\left[\operatorname{Gal}\left(F_{\infty} / F\right)\right]\right]$ modules. Let $T^{*}$ be the Tate dual of $T$. The Dieudonné module associated to $T^{*}$ is the dual of the Dieudonné module of $T$ and we denote by $\underline{I^{c}}$ the "subbasis" dual to $\underline{I}$. We prove: 
Theorem (Theorem 2.1) Assume that the Pontryagin dual of both $\operatorname{Sel}_{\underline{I}}\left(T / F_{\infty}\right)$ and $\operatorname{Sel}_{\underline{I^{c}}}\left(T^{*} / F_{\infty}\right)$ are torsion $\mathbf{Z}_{p}\left[\left[\operatorname{Gal}\left(F_{\infty} / F\right)\right]\right]$-modules, then $\operatorname{Sel}_{\underline{I}}\left(T / F_{\infty}\right)$ has no proper sub-Z ${ }_{p}\left[\left[\mathrm{Gal}\left(F_{\infty} / F\right)\right]\right]$-module of finite index.

For a good choice of basis of the Dieudonné module, one can relate the signed Selmer groups to Bloch-Kato's Selmer groups. For such a basis, assuming that the Bloch-Kato Selmer group of $T$ over $F$ is finite, our theorem above allows us to employ Greenberg's strategy in [10, Theorem 4.1] to compute the Euler-Poincaré characteristic of the signed Selmer groups. We may relate the leading term of the characteristic series of these Selmer groups to a product of Tamagawa numbers associated to the represenatation and the cardinal of the Bloch-Kato's Selmer group (see Corollary 2.10).

In the final part of the article, we study congruences of signed Selmer groups. If $E$ and $E^{\prime}$ are elliptic curves defined over $\mathbf{Q}$ with good ordinary reduction at $p$ and such that $E[p] \simeq E^{\prime}[p]$ as Galois modules, Greenberg and Vatsal [11] have studied the consequences of such a congruences in Iwasawa theory. In particular, assuming Mazur's conjecture, they proved that the $\mu$-invariant of $\operatorname{Sel}_{p}\left(E / \mathbf{Q}_{\infty}\right)$ vanishes if and only if that of $\operatorname{Sel}_{p}\left(E^{\prime} / \mathbf{Q}_{\infty}\right)$ vanishes, and that when these $\mu$-invariants do vanish, the $\lambda$-invariants of some non-primitive Selmer groups associated to $E$ and $E^{\prime}$ over $\mathbf{Q}_{\infty}$ are equal. Kim [15] generalized this result to the plus and minus Selmer groups in the supersingular case. We prove a version of this result in the setting of [5].

Theorem (Theorem 3.13) Let $T$ and $T^{\prime}$ be Galois representations to which the construction of [5] applies. Assume that $T / p T \simeq T^{\prime} / p T^{\prime}$ as Galois modules and that the Pontryagin dual of the signed Selmer groups associated to $T, T^{*}, T^{\prime}$ and $T^{\prime, *}$ are torsion $\mathbf{Z}_{p}\left[\left[\mathrm{Gal}\left(F_{\infty} / F\right)\right]\right]-$ modules. Then the $\mu$-invariant of $\operatorname{Sel}_{\underline{I}}\left(T / F_{\infty}\right)$ vanishes if and only if that of $\operatorname{Sel}_{\underline{I}}\left(T^{\prime} / F_{\infty}\right)$ vanishes. Furthemore, when these $\mu$-invariants do vanish, the $\lambda$-invariants of the $\underline{I}$-signed non-primitive Selmer groups associated to $T$ and $T^{\prime}$ over $F_{\infty}$ are equal.

The main ingredient is a result of Berger [2] who showed that the congruence $T / p T \simeq$ $T^{\prime} / p T^{\prime}$ of Galois module induces a congruence modulo $p$ on the Wach module associated to $T$ and $T^{\prime}$. This allows to keep track of the congruence through Büyükboduk and Lei's construction.

\section{Coleman maps and signed Selmer groups}

In this section, we fix notations and recall results from [5] that we shall need.

\subsection{Cyclotomic extension and Iwasawa algebra}

Choose once and for all an odd prime $p$. Let $F$ be a number field unramified at $p$. We fix $\bar{F}$ an algebraic closure of $F$ and denote by $G_{F}=\operatorname{Gal}(\bar{F} / F)$ the absolute Galois group of $F$. If $v$ is a prime of $F$, we denote by $F_{v}$ the completion of $F$ at $v, \mathcal{O}_{F_{v}}$ its ring of integers and $G_{F_{v}}$ the decomposition subgroup of $v$ in $G_{F}$. Let $\mu_{p^{n}}$ be the group of $p^{n}$ th roots of unity for every $n \geqslant 1$ and $\mu_{p^{\infty}}=\cup_{n \geqslant 1} \mu_{p^{n}}$. We set $F\left(\mu_{p^{\infty}}\right)=\cup_{n \geqslant 1} F\left(\mu_{p^{n}}\right)$ the $p^{\infty}$-cyclotomic extension of $F$ inside $\bar{F}$. For every $n \geqslant 1$, we choose a generator $\epsilon^{(n)}$ of $\mu_{p^{n}}$ with the compatibilities $\left(\epsilon^{(n+1)}\right)^{p}=\epsilon^{(n)}$, so that $\lim _{n} \epsilon^{(n)}$ is a generator of $\lim _{n} \mu_{p^{n}} \simeq \mathbf{Z}_{p}(1)$. The cyclotomic character $\chi: G_{F} \rightarrow \mathbf{Z}_{p}^{*}$ is defined by the relations $g\left(\overleftarrow{\epsilon}^{(n)}\right)=\left(\epsilon^{(n)}\right)^{\chi(g)}$ and it induces a isomorphism $\chi: \operatorname{Gal}\left(F\left(\mu_{p^{\infty}}\right) / F\right) \simeq \mathbf{Z}_{p}^{*}$. In particular, the group $\operatorname{Gal}\left(F\left(\mu_{p^{\infty}}\right) / F\right)$ 
decomposes as $\Gamma \times \Delta$ with $\Gamma \simeq \mathbf{Z}_{p}$ and $\Delta \simeq \mathbf{Z} /(p-1) \mathbf{Z}$. For every $n \geqslant 0$, we denote by $\Gamma_{n}$ the unique subgroup of $\Gamma$ of index $p^{n}$. We set $F_{\infty}=F\left(\mu_{p^{\infty}}\right)^{\Delta}$ and $F_{n}=F_{\infty}^{\Gamma_{n}}$ for every $n \geqslant 0$.

For $n \geqslant 1$, we set $\Lambda_{n}=\mathbf{Z}_{p}\left[\operatorname{Gal}\left(F\left(\mu_{p^{n}} / F\right)\right]\right.$. Let $\Lambda=\mathbf{Z}_{p}\left[\left[\operatorname{Gal}\left(F\left(\mu_{p^{\infty}}\right) / F\right)\right]\right]=$ $\lim _{n} \Lambda_{n}$ be the Iwasawa algebra of $\operatorname{Gal}\left(F\left(\mu_{p^{\infty}}\right) / F\right)$ over $\mathbf{Z}_{p}$. The above decomposition $\overleftarrow{\text { of }}_{\mathrm{Gal}}^{n}\left(F\left(\mu_{p^{\infty}}\right) / F\right)$ implies that $\Lambda=\mathbf{Z}_{p}[\Delta][[\Gamma]]$. Furthermore, we have an isomorphism $\mathbf{Z}_{p}[[\Gamma]] \simeq \mathbf{Z}_{p}[[X]]$ induced by $\gamma \mapsto X+1$ where $\gamma$ is a topological generator of $\Gamma$. For $n \geqslant 1$, let $\omega_{n}(X)=(X+1)^{p}-1$, then this isomorphism induces $\Lambda_{n} \simeq \mathbf{Z}_{p}[\Delta][X] /\left(\omega_{n}\right)$.

For a Dirichlet character $\eta$ on $\Delta$ and a $\Lambda$-module $R$, let $R^{\eta}$ be the isotypic component of $R$, which is given by $e_{\eta} \cdot R$ where $e_{\eta}=\frac{1}{|\Delta|} \sum_{\delta \in \Delta} \eta^{-1}(\delta) \delta$. Note that $R^{\eta}$ is naturally a $\mathbf{Z}_{p}[[\Gamma]]$-module. We will say that a $\Lambda$-module $R$ has rank $r$ if $R^{\eta}$ has rank $r$ over $\mathbf{Z}_{p}[[\Gamma]]$ for all characters $\eta$ on $\Delta$.

Given a finitely generated torsion $\mathbf{Z}_{p}[[\Gamma]]$-module $R$, there exists a pseudo-isomorphism (i.e. a morphism of $\mathbf{Z}_{p}[[\Gamma]]$-modules with finite kernel and cokernel)

$$
R \rightarrow \bigoplus_{i=1}^{n} \mathbf{z}_{p}[[\Gamma]] /\left(p^{l_{i}}\right) \oplus \bigoplus_{j=1}^{m} \mathbf{z}_{p}[[\Gamma]] /\left(f_{j}^{k_{j}}\right)
$$

where $f_{j} \in \mathbf{Z}_{p}[X]$ are distinguished irreducible polynomials (identifying $\mathbf{Z}_{p}[[\Gamma]]$ and $\left.\mathbf{Z}_{p}[[X]]\right)$. Furthermore, the ideals $\left(p^{l_{j}}\right)$ and $\left(f_{j}^{k_{j}}\right)$ are uniquely determined by $R$ up to ordering. The characteristic ideal of $R$ is then defined by $\prod_{i, j}\left(p^{l_{i}}\right) \cdot\left(f_{j}^{k_{j}}\right) \subset \mathbf{Z}_{p}[[\Gamma]]$. The $\mu$-invariant of $R$ is defined by $\sum_{i=1}^{n} l_{i}$ and the $\lambda$-invariant of $R$ by $\sum_{j=1}^{m} k_{j} \cdot \operatorname{deg} f_{j}$.

\subsection{Motives}

Let $\mathcal{M}$ be a motive defined over $F$ with coefficients in $\mathbf{Q}$ in the sense of [8]. We denote by $\mathcal{M}_{p}$ its $p$-adic realization and we fix $T$ a $G_{F}$-stable $\mathbf{Z}_{p}$-lattice inside $\mathcal{M}_{p}$. Let $g=$ $\operatorname{dim}_{\mathbf{Q}_{p}}\left(\operatorname{Ind}_{F}^{\mathbf{Q}} \mathcal{M}_{p}\right)$ and $g_{+}=\operatorname{dim}_{\mathbf{Q}_{p}}\left(\operatorname{Ind}_{F}^{\mathbf{Q}} \mathcal{M}_{p}\right)^{+}$the dimension of the +1 -eigenspace under the action of a fixed complex conjugation on $\operatorname{Ind}_{F}^{\mathbf{Q}} \mathcal{M}_{p}$. We set $g_{-}=g-g_{+}$. For every prime $v$ of $F$ dividing $p$, let $g_{v}=\operatorname{dim}_{\mathbf{Q}_{p}}\left(\operatorname{Ind}_{F_{v}} \mathbf{M}_{p}\right)$. We have $g=\sum_{v \mid p} g_{v}$.

We will assume that, for every prime $v$ of $F$ dividing $p$,

(H.-T.) the Hodge-Tate weights of $\mathcal{M}_{p}$, as a $G_{F_{v}}$-representation, are in [0,1],

(Cryst.) the $G_{F_{v}}$-representation $\mathcal{M}_{p}$ is crystalline,

(Tors.) the Galois cohomology groups $\mathrm{H}^{0}\left(F_{v}, T / p T\right)$ and $\mathrm{H}^{2}\left(F_{v}, T / p T\right)$ are trivial.

We denote by $T^{*}=\operatorname{Hom}\left(T, \mathbf{Z}_{p}(1)\right)$ the Tate dual of $T$ and we set

$$
M=T \otimes \mathbf{Q}_{p} / \mathbf{Z}_{p}, \quad \text { and }, \quad M^{*}=T^{*} \otimes \mathbf{Q}_{p} / \mathbf{Z}_{p} .
$$

We remark that the dual of $\mathcal{M}$, which we denote by $\mathcal{M}^{*}$, satisfies the hypothesis (Cryst.) and (H.-T.), and $T^{*}$, which is a $G_{F}$-stable $\mathbf{Z}_{p}$-lattice inside its $p$-adic realization $\mathcal{M}_{p}^{*}$, satisfies (Tors.).

We also fix $\Sigma$ a finite set of primes of $F$ containing the primes dividing $p$, the archimedean primes and the primes of ramification of $M^{*}$. Let $F_{\Sigma}$ be the maximal extension of $F$ unramified outside $\Sigma$, so that $M^{*}$ is a $\operatorname{Gal}\left(F_{\Sigma} / F\right)$-module. We remark that $F\left(\mu_{p} \infty\right) \subseteq F_{\Sigma}$ since only primes above $p$ and $\infty$ can be ramified in $F\left(\mu_{p^{\infty}}\right)$. If $F^{\prime}$ is an extension of $F$ in $F\left(\mu_{p^{\infty}}\right)$, we will say by abuse that a prime of $F^{\prime}$ lies in $\Sigma$ if it divides a prime of $F$ which is in $\Sigma$. 


\subsection{Dieudonné modules}

If $v$ is a prime of $F$ dividing $p$, let $\mathbf{D}_{\text {cris, } v}(T)$ be the Dieudonné module associated to $T$ considered as a $G_{F_{v}}$-representation [2, Définition V.1.1]. Then $\mathbf{D}_{\text {cris, } v}(T)$ is a free $\mathcal{O}_{F_{v}}{ }^{-}$ module of rank $\operatorname{dim}_{\mathbf{Q}_{p}} \mathcal{M}_{p}$ equipped with a filtration of $\mathcal{O}_{F_{v}}$-modules $\left(\text { Fil }{ }^{i} \mathbf{D}_{\text {cris }, v}(T)\right)_{i \in \mathbf{Z}}$ such that

$$
\text { Fil }^{i} \mathbf{D}_{\text {cris }, v}(T)= \begin{cases}0 & \text { for } i \geqslant 1, \\ \mathbf{D}_{\text {cris }, v}(T) & \text { for } i \leqslant-1 .\end{cases}
$$

Furthermore, $\mathbf{D}_{\text {cris }, v}\left(\mathcal{M}_{p}\right):=\mathbf{D}_{\text {cris }, v}(T) \otimes \mathbf{Q}_{p}$ is the usual Fontaine's filtered $\phi$-module associated to $\mathcal{M}_{p}$.

We will assume that

(Fil.) $\sum_{v \mid p} \operatorname{dim}_{\mathbf{Q}_{p}} \operatorname{Fil}^{0} \mathbf{D}_{\text {cris }, v}(T) \otimes \mathbf{Q}_{p}=g_{-}$,

(Slopes) the slopes of $\phi$ are in ] - 1, 0[.

We may choose $\left\{u_{1}, \ldots, u_{g_{v}}\right\}$ a $\mathbf{Z}_{p}$-basis of $\mathbf{D}_{\text {cris }, v}(T)$ such that $\left\{u_{1}, \ldots, u_{d_{v}}\right\}$ is a basis for $\mathrm{Fil}^{0} \mathbf{D}_{\text {cris }, v}(T)$ for some $d_{v}$. We call such a basis a Hodge-compatible basis and fix one for the rest of the paper. Then, from our hypotheses, the matrix of the crystalline Frobenius $\phi$ with respect to this basis is of the form

$$
C_{\phi, v}=C_{v}\left(\begin{array}{c|c}
I_{d_{v}} & 0 \\
\hline 0 \mid \frac{1}{p} I_{g_{v}-d_{v}}
\end{array}\right)
$$

where $C_{v} \in \mathrm{GL}_{g_{v}}\left(\mathbf{Z}_{p}\right)$ and $I_{n}$ is the identity matrix of size $n$.

Let $\mathbf{D}_{\text {cris }, v}\left(T^{*}\right)$ be the Dieudonné module associated to $T^{*}$. There is a natural pairing

$$
\mathbf{D}_{\text {cris }, v}(T) \times \mathbf{D}_{\text {cris }, v}\left(T^{*}\right) \rightarrow \mathbf{D}_{\text {cris }, v}\left(\mathbf{Z}_{p}(1)\right) \simeq \mathbf{Z}_{p},
$$

with respect to which Fil ${ }^{i} \mathbf{D}_{\text {cris }, v}\left(T^{*}\right)$ is the orthogonal complement of Fil ${ }^{-i} \mathbf{D}_{\text {cris, } v}(T)$ and $\phi^{-1}$ is the dual of $p \phi$. In particular, $\mathbf{D}_{\text {cris, } v}\left(T^{*}\right)$ also satisfies the hypotheses (Fil.) and (Slopes).

Example 1.1 Let $A$ be an abelian variety defined over $F$ with good supersingular reduction at every prime dividing $p$. Let $T_{p}(A)=\lim _{n} A\left[p^{n}\right]$ be the $p$-adic Tate module of $A$ and

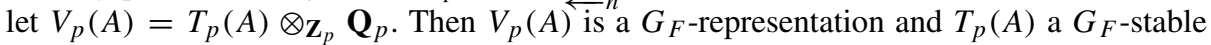
$\mathbf{Z}_{p}$-lattice of $V_{p}(A)$ which satisfy all the hypotheses (Crys.), (H.-T.), (Tors.), (Fil.) and (Slopes).

\subsection{Decomposition of Perrin-Riou's big logarithm map}

Let $v$ be a prime of $F$ dividing $p$. For $i \geqslant 0$, the projective limit of the Galois cohomology groups $\mathrm{H}^{i}\left(F_{v}\left(\mu_{p^{n}}\right), T\right)$ relative to the corestriction maps is denoted by $\mathrm{H}_{\mathrm{Iw}}^{i}\left(F_{v}, T\right)$. Recall that $\mathrm{H}_{\mathrm{Iw}}^{1}\left(F_{v}, T\right)$ is a $\Lambda$-module of rank $g_{v}$ [26, Proposition A.2.3 ii)].

We set $\mathcal{H}=\mathbf{Q}_{p}[\Delta] \otimes_{\mathbf{Q}_{p}} \mathcal{H}(\Gamma)$ where $\mathcal{H}(\Gamma)$ is the set of elements $f(\gamma-1)$ with $\gamma \in \Gamma$ and $f(X) \in \mathbf{Q}_{p}[[X]]$ is convergent on the $p$-adic open unit disk. Perrin-Riou's big logarithm map is a $\Lambda$-homomorphism [25]

$$
\mathcal{L}_{T, v}: \mathrm{H}_{\mathrm{IW}}^{1}\left(F_{v}, T\right) \rightarrow \mathcal{H} \otimes \mathbf{z}_{p} \mathbf{D}_{\text {cris }, v}(T)
$$

which interpolates Kato's dual exponential maps [13, II Sect. 1.2]

$$
\exp _{n}^{*}: \mathrm{H}^{1}\left(F_{v}\left(\mu_{p^{n}}\right), T\right) \rightarrow F_{v}\left(\mu_{p^{n}}\right) \otimes \mathbf{z}_{p} \mathbf{D}_{\text {cris }, v}(T) .
$$


As in [5], we may define for $n \geqslant 1$,

$$
C_{v, n}=\left(\begin{array}{c|c}
I_{d_{v}} & 0 \\
\hline 0 & \Phi_{p^{n}}(1+X) I_{g_{v}}-d_{v}
\end{array}\right) C_{v}^{-1} \text { and } M_{v, n}=\left(C_{\phi, v}\right)^{n+1} C_{v, n} \cdots C_{1},
$$

where $\Phi_{p^{n}}$ is the $p^{n}$ th cyclotomic polynomial. By Proposition 2.5 in op. cit., the sequence $\left(M_{v, n}\right)_{n \geqslant 1}$ converges to some $g_{v} \times g_{v}$ logarithmic matrix over $\mathcal{H}$, which we denote by $M_{v}$. This allows to decompose $\mathcal{L}_{T, v}$ into

$$
\mathcal{L}_{T, v}=\left(u_{1}, \ldots, u_{g_{v}}\right) \cdot M_{v} \cdot\left(\begin{array}{c}
\mathrm{Col}_{T, v, 1} \\
\vdots \\
\mathrm{Col}_{T, v, g_{v}}
\end{array}\right)
$$

where $\mathrm{Col}_{T, v, i}, i \in\left\{1, \ldots, g_{v}\right\}$ are $\Lambda$-homomorphisms from $\mathrm{H}_{\mathrm{IW}}^{1}\left(F_{v}, T\right)$ to $\Lambda$. More details on the decomposition (4) are given in Sect. 3.2.

\subsection{Signed Coleman maps}

Let $I_{v}$ be a subset of $\left\{1, \ldots, g_{v}\right\}$. We set

$$
\mathrm{Col}_{T, I_{v}}: \mathrm{H}_{\mathrm{IW}}^{1}\left(F_{v}, T\right) \rightarrow \bigoplus_{i=1}^{\left|I_{v}\right|} \Lambda, \quad \mathbf{z} \mapsto\left(\operatorname{Col}_{T, v, i}(\mathbf{z})\right)_{i \in I_{v}} .
$$

These maps are called signed Coleman maps. We recall results about them that we shall need.

\section{Lemma 1.2 ([5, Proposition 2.20, Lemma 3.22])}

1. For any character $\eta$ on $\Delta$, the $\eta$-isotypic component of the image of the signed Coleman map $\operatorname{Im~} \mathrm{Col}_{T, I_{v}}^{\eta}$ is a $\mathbf{Z}_{p}[[\Gamma]]$-module of rank $\left|I_{v}\right|$ contained in a free $\mathbf{Z}_{p}[[\Gamma]]$-module with finite index.

2. The $\Lambda$-module $\operatorname{Ker}^{\mathrm{Col}_{T} I_{v}}$ is free of rank $g_{v}-\left|I_{v}\right|$.

Let

$$
\langle\cdot, \cdot\rangle_{n}: \mathrm{H}^{1}\left(F_{v}\left(\mu_{p^{n}}\right), T\right) \times \mathrm{H}^{1}\left(F_{v}\left(\mu_{p^{n}}\right), T^{*}\right) \rightarrow \mathrm{H}^{2}\left(F_{v}\left(\mu_{p^{n}}\right), \mathbf{Z}_{p}(1)\right) \simeq \mathbf{Z}_{p},
$$

be Tate's local pairing. If $x=\left(x_{n}\right)_{n}$ and $y=\left(y_{n}\right)_{n}$ are elements of $\mathrm{H}_{\mathrm{IW}}^{1}\left(F_{v}, T\right)$ and $\mathrm{H}_{\mathrm{IW}}^{1}\left(F_{v}, T^{*}\right)$ then the elements

$$
\sum_{\sigma \in \operatorname{Gal}\left(F_{v}\left(\mu_{p^{n}}\right) / F_{v}\right)}\left\langle x_{n}, \sigma\left(y_{n}\right)\right\rangle \sigma \in \mathbf{Z}_{p}\left[\operatorname{Gal}\left(F_{v}\left(\mu_{p^{n}}\right) / F_{v}\right)\right],
$$

are compatible under the natural projection maps

$$
\mathbf{Z}_{p}\left[\operatorname{Gal}\left(F_{v}\left(\mu_{p^{n+1}}\right) / F_{v}\right)\right] \rightarrow \mathbf{Z}_{p}\left[\operatorname{Gal}\left(F_{v}\left(\mu_{p^{n}}\right) / F_{v}\right)\right]
$$

thus, they define an element in $\Lambda$. This defines Perrin-Riou's pairing

$$
\mathrm{H}_{\mathrm{IW}}^{1}\left(F_{v}, T\right) \times \mathrm{H}_{\mathrm{Iw}}^{1}\left(F_{v}, T^{*}\right) \rightarrow \Lambda .
$$

Since all our hypotheses (Crys.), (H.-T.), (Tors.), (Fil.) and (Slopes) are satisfied by $\mathcal{M}^{*}$ and $T^{*}$, we carry out all of the constructions of paragraph 1.4 for $T^{*}$ with respect to the dual basis of our fixed basis $\left\{u_{1}, \ldots, u_{g_{v}}\right\}$ for the pairing (2) and similarly define signed Coleman maps for $T^{*}$.

Then, we have the following relation. 
Lemma 1.3 ([21, Lemma 3.2]) Let $I_{v}$ be a subset of $\left\{1, \ldots, g_{v}\right\}$ and $I_{v}^{c}$ its complement. Then $\operatorname{Ker} \mathrm{Col}_{T, I_{v}}$ is the orthogonal complement of $\mathrm{Ker}^{\mathrm{Col}_{T}^{*}, I_{v}^{c}}$ relative to Perrin-Riou's pairing (5).

Remark 1.4 In [21], there is an additional hypothesis that $g_{+}=g_{-}$and $F$ is abelian over $\mathbf{Q}$ with degree prime to $p$. However, the proof of Lemma 3.2 in op. cit. applies in the setting considered in the present article in verbatim.

\subsection{Signed Selmer groups}

Let $\underline{I}=\left(I_{v}\right)_{v \mid p}$ be a tuple of sets indexed by the primes $v$ of $F$ dividing $p$ and where each $I_{v}$ is a subset of $\left\{1, \ldots, g_{v}\right\}$.

Tate's local pairing

$$
\mathrm{H}^{1}\left(F_{v}\left(\mu_{p^{n}}\right), M^{*}\right) \times \mathrm{H}^{1}\left(F_{v}\left(\mu_{p^{n}}\right), T\right) \rightarrow \mathrm{H}^{2}\left(F_{v}\left(\mu_{p^{n}}\right), \mu_{p^{\infty}}\right) \simeq \mathbf{Q}_{p} / \mathbf{Z}_{p}
$$

passes to the limit relative to restriction and corestriction and defines a pairing

$$
\mathrm{H}^{1}\left(F_{v}\left(\mu_{p^{\infty}}\right), M^{*}\right) \times \mathrm{H}_{\mathrm{Iw}}^{1}\left(F_{v}, T\right) \rightarrow \mathbf{Q}_{p} / \mathbf{Z}_{p} .
$$

Definition 1.5 We define $\mathrm{H}_{I_{v}}^{1}\left(F_{v}\left(\mu_{p^{\infty}}\right), M^{*}\right) \subseteq \mathrm{H}^{1}\left(F_{v}\left(\mu_{p^{\infty}}\right), M^{*}\right)$ as the orthogonal complement of $\operatorname{Ker~} \operatorname{Col}_{T, I_{v}}$ under the pairing (6).

The assumption $\mathrm{H}^{2}\left(F_{v}, T / p T\right)=0$ (Tors.) implies by Tate's duality that $\mathrm{H}^{0}\left(F_{v}, M^{*}\right)=$ 0 , thus $\mathrm{H}^{0}\left(F_{v, \infty}, M^{*}\right)=0$ since $\operatorname{Gal}\left(F_{v, \infty} / F_{v}\right) \simeq \mathbf{Z}_{p}$ is a pro- $p$-group. In particular, by the inflation-restriction exact sequence, we have

$$
\mathrm{H}^{1}\left(F_{v, \infty}, M^{*}\right) \simeq \mathrm{H}^{1}\left(F_{v}\left(\mu_{p^{\infty}}\right), M^{*}\right)^{\Delta}
$$

since the order of $\Delta$ is $p-1$ and $\mathrm{H}^{0}\left(F_{v}\left(\mu_{p^{\infty}}\right), M^{*}\right)$ is finite of order a power of $p$, and for $n \geqslant 0$, we have

$$
\mathrm{H}^{1}\left(F_{v, n}, M^{*}\right) \simeq \mathrm{H}^{1}\left(F_{v, \infty}, M^{*}\right)^{\Gamma_{n}}
$$

We set

$$
\mathrm{H}_{I_{v}}^{1}\left(F_{v, \infty}, M^{*}\right)=\mathrm{H}_{I_{v}}^{1}\left(F_{v}\left(\mu_{p^{\infty}}\right), M^{*}\right)^{\Delta} \text { and } \mathrm{H}_{I_{v}}^{1}\left(F_{v, n}, M^{*}\right)=\mathrm{H}_{I_{v}}^{1}\left(F_{v, \infty}, M^{*}\right)^{\Gamma_{n}} .
$$

We also have signed Coleman maps for $T^{*}$. For $n \geqslant 0$, let $\left(\operatorname{Ker} \operatorname{Col}_{T^{*}, I_{v}^{c}}\right)_{n}$ be the image

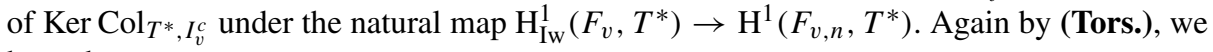
have the exact sequence

$$
0 \rightarrow \mathrm{H}^{1}\left(F_{v, n}, T^{*}\right) \stackrel{i_{n}}{\rightarrow} \mathrm{H}^{1}\left(F_{v, n}, \mathcal{M}_{p}^{*}\right) \stackrel{\pi_{n}}{\rightarrow} \mathrm{H}^{1}\left(F_{v, n}, M^{*}\right) \rightarrow 0 .
$$

The image of $\left(\operatorname{Ker} \mathrm{Col}_{T^{*}, I_{v}^{c}}\right)_{n}$ under $i_{n}$ generates a $\mathbf{Q}_{p}$-vector space in $\mathrm{H}^{1}\left(F_{v, n}, \mathcal{M}_{p}^{*}\right)$, and we denote by $\overline{\left(\operatorname{Ker~Col}_{\left.T^{*}, I_{v}^{c}\right)_{n}}\right.}$ the image of this $\mathbf{Q}_{p}$-vector space in $\mathrm{H}^{1}\left(F_{v, n}, M^{*}\right)$ under $\pi_{n}$.

Lemma 1.6 For any $n \geqslant 0, \overline{\left(\operatorname{Ker}^{-C} T^{*}, I_{v}^{c}\right)_{n}}$ is the orthogonal complement of $\left(\operatorname{Ker} \operatorname{Col}_{T, I_{v}}\right)_{n}$ under Tate's local pairing

$$
\mathrm{H}^{1}\left(F_{v, n}, M^{*}\right) \times \mathrm{H}^{1}\left(F_{v, n}, T\right) \rightarrow \mathrm{H}^{2}\left(F_{v, n}, \mu_{p} \infty\right) \simeq \mathbf{Q}_{p} / \mathbf{Z}_{p} .
$$

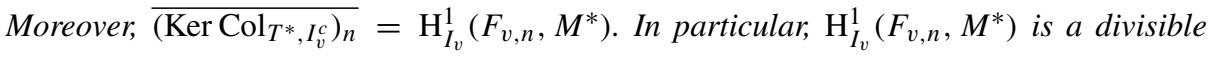
group. 
Proof By Lemma 1.3 and bilinearity of Tate's pairing, the orthogonal complement of $\left(\operatorname{Ker~Col}_{T, I_{v}}\right)_{n}$ under Tate's pairing contains $\overline{\left(\operatorname{Ker~Col}_{\left.T^{*}, I_{v}^{c}\right)_{n}}\right.}$. The reverse inclusion follows from the exactness of the sequence (7). As already remarked, by (Tors.), one has $\mathrm{H}^{1}\left(F_{v, n}, M^{*}\right)=\mathrm{H}^{1}\left(F_{v}\left(\mu_{p^{\infty}}\right), M^{*}\right)^{\Gamma_{n} \times \Delta}$ and by duality $\mathrm{H}_{\mathrm{IW}}^{1}\left(F_{v}, T\right)_{\Gamma_{n} \times \Delta}=\mathrm{H}^{1}\left(F_{v, n}, T\right)$. It follows that $\overline{\left(\operatorname{Ker~Col}_{\left.T^{*}, I_{v}^{c}\right)_{n}}\right.}=\mathrm{H}_{I_{v}}^{1}\left(F_{v, n}, M^{*}\right)$.

Let $w$ be a prime of $F$ not dividing $p$ and let $K$ be a finite extension of $F_{w}$. Define

$$
\mathrm{H}_{\mathrm{unr}}^{1}\left(K, \mathcal{M}_{p}^{*}\right)=\operatorname{Ker}\left(\mathrm{H}^{1}\left(K, \mathcal{M}_{p}^{*}\right) \rightarrow \mathrm{H}^{1}\left(K_{\mathrm{unr}}, \mathcal{M}_{p}^{*}\right)\right)
$$

where $K_{\text {unr }}$ the maximal unramified extension of $K$. Let $\mathrm{H}_{\mathrm{unr}}^{1}\left(K, M^{*}\right)$ be the image of $\mathrm{H}_{\mathrm{unr}}^{1}\left(K, \mathcal{M}_{p}^{*}\right)$ under the natural map

$$
\mathrm{H}^{1}\left(K, \mathcal{M}_{p}^{*}\right) \rightarrow \mathrm{H}^{1}\left(K, M^{*}\right)
$$

and $\mathrm{H}_{\mathrm{unr}}^{1}\left(K, T^{*}\right)$ the inverse image of $\mathrm{H}_{\mathrm{unr}}^{1}\left(K, \mathcal{M}_{p}^{*}\right)$ under

$$
\mathrm{H}^{1}\left(K, T^{*}\right) \rightarrow \mathrm{H}^{1}\left(K, \mathcal{M}_{p}^{*}\right) .
$$

We remark that $\mathrm{H}_{\mathrm{unr}}^{1}\left(K, M^{*}\right)$ is divisible by definition and recall that it is the orthogonal complement of $\mathrm{H}_{\mathrm{unr}}^{1}(K, T)$ under Tate's local pairing (see [3, Proposition 3.8]). If $K^{\prime}$ is an infinite algebraic extension of $F_{w}$, we define the subgroup

$$
\mathrm{H}_{\mathrm{unr}}^{1}\left(K^{\prime}, M^{*}\right)=\underset{K}{\lim } \mathrm{H}_{\mathrm{unr}}^{1}\left(K, M^{*}\right) \subset \mathrm{H}^{1}\left(K^{\prime}, M^{*}\right)
$$

where the limit runs through the finite extensions $K$ of $F_{w}$ contained in $K^{\prime}$ and is taken with respect to the restriction maps.

Let $F^{\prime}$ be one of $F\left(\mu_{p^{\infty}}\right), F_{\infty}$ or $F_{n}$ for some $n \geqslant 0$. We set

$$
\mathcal{P}_{\Sigma, \underline{I}}\left(M^{*} / F^{\prime}\right)=\prod_{w \in \Sigma, w \nmid p} \frac{\mathrm{H}^{1}\left(F_{w}^{\prime}, M^{*}\right)}{\mathrm{H}_{\mathrm{unr}}^{1}\left(F_{w}^{\prime}, M^{*}\right)} \times \prod_{w \mid p} \frac{\mathrm{H}^{1}\left(F_{w}^{\prime}, M^{*}\right)}{\mathrm{H}_{I_{v}}^{1}\left(F_{w}^{\prime}, M^{*}\right)} .
$$

Definition 1.7 Let $F^{\prime}$ be $F\left(\mu_{p^{\infty}}\right), F_{\infty}$, or $F_{n}$ for some $n \geqslant 0$. The $\underline{I}$-Selmer group of $M^{*}$ over $F^{\prime}$ is defined by

$$
\operatorname{Sel}_{\underline{I}}\left(M^{*} / F^{\prime}\right)=\operatorname{Ker}\left(\mathrm{H}^{1}\left(F_{\Sigma} / F^{\prime}, M^{*}\right) \rightarrow \mathcal{P}_{\Sigma, \underline{I}}\left(M^{*} / F^{\prime}\right)\right)
$$

where the map is the composition of localization at each $w \in \Sigma$ followed by the projection in the appropriate quotient.

Let $\mathcal{I}_{p}$ be the set of tuples $\underline{I}=\left(I_{v}\right)_{v \mid p}$ indexed by the primes $v$ of $F$ dividing $p$ and where each $I_{v}$ is a subset of $\left\{1, \ldots, g_{v}\right\}$ and such that $\sum_{v \mid p}\left|I_{v}\right|=g_{-}$. From observations about the expected $\Lambda$-corank of the Selmer group of a supersingular abelian variety, Büyükboduk and Lei have made the following conjecture [5, Remark 3.27].

Conjecture 1.8 For any $\underline{I} \in \mathcal{I}_{p}$ and any even Dirichlet character $\eta$ on $\Delta, \operatorname{Sel}_{\underline{I}}\left(M^{*} / F\left(\mu_{p^{\infty}}\right)\right)^{\eta}$ is a cotorsion $\mathbf{Z}_{p}[[\Gamma]]$-module (i.e. its Pontryagin dual is a torsion $\mathbf{Z}_{p}[[\Gamma]]$-module).

Remark 1.9 When $F=\mathbf{Q}$ and $\mathcal{M}$ is the Tate module of a supersingular elliptic curve with $a_{p}=0$, for a good choice of basis of the Dieudonné module, the signed Selmer groups with $\underline{I} \in \mathcal{I}_{p}$ coincide with Kobayashi plus and minus Selmer groups [19] (see [5, Appendix 4]). Conjecture 1.8 is known in that case $o p$. cit.. Furthermore, Sprung [6] as well as Lei, Loeffler and Zerbes [20] have proved that this conjecture holds in cases of $p$-supersingular elliptic curves with $a_{p} \neq 0$ and $p$-non-ordinary eigenforms, respectively. 
Remark 1.10 The definition of the signed Selmer groups does not depend on the choice of $\Sigma$. If $\eta$ is the trivial character on $\Delta$, then $\operatorname{Sel}_{\underline{I}}\left(M^{*} / F\left(\mu_{p^{\infty}}\right)\right)^{\eta} \simeq \operatorname{Sel}_{\underline{I}}\left(M^{*} / F_{\infty}\right)$. It follows from the definition that, for any $\underline{I}$, the Pontryagin dual of $\operatorname{Sel}_{I}\left(M^{*} / F_{\infty}\right)$ is a finitely generated $\mathbf{Z}_{p}[[\Gamma]]$-module since $\mathrm{H}^{1}\left(F_{\Sigma} / F_{\infty}, M^{*}\right)$ is [9, Proposition 3]. In the remainder of this article, we study these Selmer groups.

In the next section, we shall need twisted signed Selmer groups. Let us explain now what they are. For $s \in \mathbf{Z}$, we set $M_{s}^{*}=M^{*} \otimes \chi_{\mid \Gamma}^{s}$ where $\chi_{\mid \Gamma}: \Gamma \simeq \mathbf{Z}_{p}$. As a $\operatorname{Gal}\left(\bar{F} / F_{\infty}\right)$ module, $M_{s}^{*}=M^{*}$, thus $\mathrm{H}^{1}\left(F_{\infty}, M_{s}^{*}\right)=\mathrm{H}^{1}\left(F_{\infty}, M^{*}\right) \otimes \chi_{\mid \Gamma}^{s}$ and for a prime $v$ of $F$, $\mathrm{H}^{1}\left(F_{v, \infty}, M_{s}^{*}\right)=\mathrm{H}^{1}\left(F_{v, \infty}, M^{*}\right) \otimes \chi_{\mid \Gamma}^{s}$ and $\mathrm{H}^{0}\left(F_{v, \infty}, M_{s}^{*}\right)=0$. At primes dividing $p$, we set

$$
\mathrm{H}_{I_{v}}^{1}\left(F_{v, \infty}, M_{s}^{*}\right)=\mathrm{H}_{I_{v}}^{1}\left(F_{v, \infty}, M^{*}\right) \otimes \chi_{\mid \Gamma}^{s} .
$$

Therefore, for $F^{\prime}$ being $F_{\infty}$ or $F_{n}$ for some $n \geqslant 0$, we can define twisted $\underline{I}$-Selmer groups $\operatorname{Sel}_{\underline{I}}\left(M_{s}^{*} / F^{\prime}\right)$ as above with local condition at $p$ induced by $\mathrm{H}_{I_{v}}^{1}\left(F_{v, \infty}, M_{s}^{*}\right)$. We remark that $\operatorname{Sel}_{\underline{I}}\left(M_{s}^{*} / F_{\infty}\right) \simeq \operatorname{Sel}_{\underline{I}}\left(M^{*} / F_{\infty}\right) \otimes \chi_{\mid \Gamma}^{S}$ as $\mathbf{Z}_{p}[[\Gamma]]$-modules.

Similarly, we can define signed Selmer groups for $M$ using the signed Coleman maps $\mathrm{Col}_{T^{*}, I_{v}}$, as well as twisted signed Selmer groups for $M$ as above. We remark that if $\underline{I}$ is an element of $\mathcal{I}_{p}$, then $\underline{I}^{c}=\left(I_{v}^{c}\right)_{v \mid p}$ satisfies $\sum_{v \mid p} I_{v}^{c}=g-g_{-}=g_{+}=\operatorname{dim}_{\mathbf{Q}_{p}}\left(\operatorname{Ind}_{F}^{\mathbf{Q}} \overline{\mathcal{M}}_{p}^{*}\right)^{-}$. In particular, Conjecture 1.8 is expected to hold for the signed Selmer groups of $M$.

\subsection{Bloch-Kato's Selmer groups}

Let $n \geqslant 0$ and $w$ be a prime of $F_{n}$ dividing $p$. We recall that Bloch and Kato [3] defined the $\mathbf{Q}_{p}$-subspace of $\mathrm{H}^{1}\left(F_{n, w}, \mathcal{M}_{p}^{*}\right)$

$$
\mathrm{H}_{f}^{1}\left(F_{n, w}, \mathcal{M}_{p}^{*}\right)=\operatorname{Ker}\left(\mathrm{H}^{1}\left(F_{n, w}, \mathcal{M}_{p}^{*}\right) \rightarrow \mathrm{H}^{1}\left(F_{n, w}, \mathbf{B}_{\mathrm{cris}} \otimes \mathcal{M}_{p}^{*}\right)\right)
$$

where $\mathbf{B}_{\text {cris }}$ is Fontaine's ring of crystalline periods [7]. Let $\mathrm{H}_{f}^{1}\left(F_{n, w}, M^{*}\right)$ be the image of $\mathrm{H}_{f}^{1}\left(F_{n, w}, \mathcal{M}_{p}^{*}\right)$ under the natural map

$$
\mathrm{H}^{1}\left(F_{n, w}, \mathcal{M}_{p}^{*}\right) \rightarrow \mathrm{H}^{1}\left(F_{n, w}, M^{*}\right) .
$$

We set

$$
\mathcal{P}_{\Sigma, f}\left(M^{*} / F_{n}\right)=\prod_{w \in \Sigma, w \nmid p} \frac{\mathrm{H}^{1}\left(F_{n, w}, M^{*}\right)}{\mathrm{H}_{\mathrm{unr}}^{1}\left(F_{n, w}, M^{*}\right)} \times \prod_{w \mid p} \frac{\mathrm{H}^{1}\left(F_{n, w}, M^{*}\right)}{\mathrm{H}_{f}^{1}\left(F_{n, w}, M^{*}\right)} .
$$

Then, the Bloch-Kato's Selmer group of $M^{*}$ over $F_{n}$ is defined by

$$
\operatorname{Sel}_{\mathrm{BK}}\left(M^{*} / F_{n}\right)=\operatorname{Ker}\left(\mathrm{H}^{1}\left(F_{\Sigma} / F_{n}, M^{*}\right) \rightarrow \mathcal{P}_{\Sigma, f}\left(M^{*} / F_{n}\right)\right)
$$

and we set $\operatorname{Sel}_{\mathrm{BK}}\left(M^{*} / F_{\infty}\right)=\lim _{n} \operatorname{Sel}_{\mathrm{BK}}\left(M^{*} / F_{n}\right)$.

Recall that the definition of the signed Coleman maps and thus of the signed Selmer groups depends on a choice of Hodge-compatible basis of $\oplus_{v \mid p} \mathbf{D}_{\text {cris, } v}(T)$.

Lemma 1.11 ([4, Lemma 8.1]) There exists a Hodge-compatible basis of $\oplus_{v \mid p} \mathbf{D}_{\mathrm{cris}, v}(T)$ such that for any $\underline{I} \in \mathcal{I}_{p}$

$$
\mathrm{H}_{f}^{1}\left(F_{v}, M^{*}\right)=\mathrm{H}_{I_{v}}^{1}\left(F_{v}, M^{*}\right)
$$


In particular, for such a basis,

$$
\operatorname{Sel}_{\mathrm{BK}}\left(M^{*} / F\right)=\operatorname{Sel}_{\underline{I}}\left(M^{*} / F\right) .
$$

The basis of the lemma is a strongly admissible basis in the sense of [5, Definition 3.2].

\section{Submodules of finite index}

We keep the notation of the previous section. Let $\underline{I}=\left(I_{v}\right)_{v \mid p} \in \mathcal{I}_{p}$ and set $\underline{I}^{c}=\left(I_{v}^{c}\right)_{v \mid p}$. The main goal of this section is to prove the following theorem.

Theorem 2.1 Assume that $\operatorname{Sel}_{\underline{I}}\left(M^{*} / F_{\infty}\right)$ and $\operatorname{Sel}_{I^{c}}\left(M / F_{\infty}\right)$ are cotorsion $\mathbf{Z}_{p}[[\Gamma]]$-modules. Then $\operatorname{Sel}_{\underline{I}}\left(M^{*} / F_{\infty}\right)$ has no proper sub- $\mathbf{Z}_{p}[[\Gamma]]$-modules of finite index.

Remark 2.2 Under the additional hypothesis that $F$ is abelian over $\mathbf{Q}$ with degree prime to $p$ and that $g_{+}=g_{-}$, an algebraic functional equation relating $\operatorname{Sel}_{\underline{I}}\left(M^{*} / F_{\infty}\right)$ and $\operatorname{Sel}_{\underline{I}^{c}}\left(M / F_{\infty}\right)$ has been proved in [21]. In this situation, if one of these $\mathbf{Z}_{p}[[\Gamma]]$-modules is a cotorsion $\mathbf{Z}_{p}[[\Gamma]]$-module, then they both are.

\subsection{The proof of Theorem 2.1}

We begin with a "control theorem" for these signed Selmer groups.

Lemma 2.3 For all but finitely many $s \in \mathbf{Z}$, the kernel and cokernel of the restriction map

$$
\operatorname{Sel}_{\underline{I}^{c}}\left(M_{S} / F_{n}\right) \rightarrow \operatorname{Sel}_{\underline{\underline{I}}^{c}}\left(M_{S} / F_{\infty}\right)^{\Gamma_{n}}
$$

are finite of bounded orders as $n$ varies.

Proof The diagram

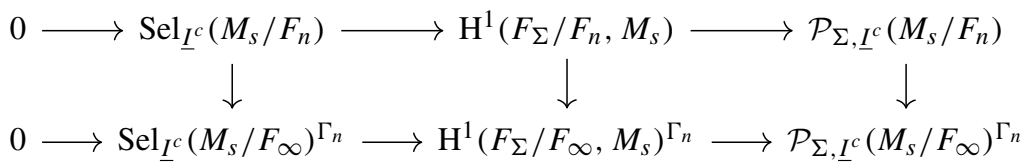

is commutative.

By (Tors.), $\mathrm{H}^{0}\left(F_{v, \infty}, M_{s}\right)=0$ where $v$ is any prime of $F$ dividing $p$, thus the central map is an isomorphism by the inflation-restriction exact sequence.

We now study the kernel of the rightmost vertical map. For a prime $v$ of $F$ dividing $p$, the diagram

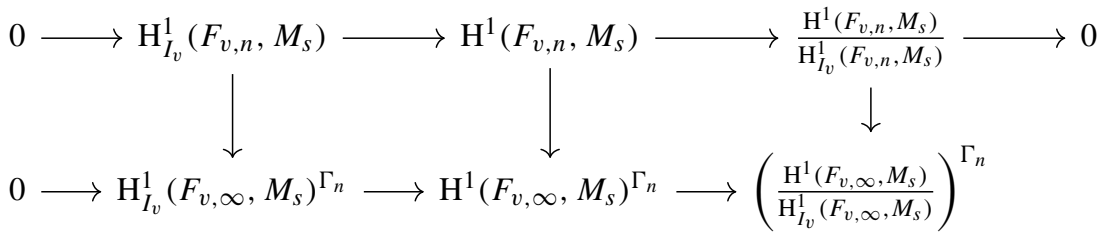

is commutative. The central vertical map is an isomorphism by the inflation-restriction exact sequence and the left-most vertical one is an isomorphism by definition, thus it follows from the snake lemma applied to the diagram (9) that the map 


$$
\frac{\mathrm{H}^{1}\left(F_{v, n}, M_{s}\right)}{\mathrm{H}_{I_{v}}^{1}\left(F_{v, n}, M_{s}\right)} \rightarrow\left(\frac{\mathrm{H}^{1}\left(F_{v, \infty}, M_{s}\right)}{\mathrm{H}_{I_{v}}^{1}\left(F_{v, \infty}, M_{s}\right)}\right)^{\Gamma_{n}}
$$

is an injection.

For a prime $w$ of $F_{n}$ not dividing $p$ and a prime $w^{\prime}$ of $F_{\infty}$ above $w$, the diagram

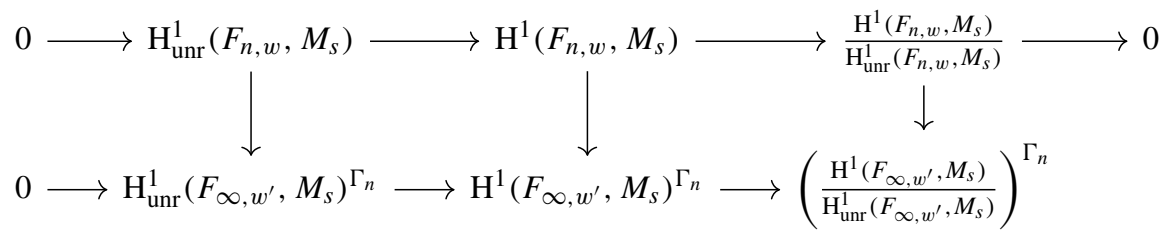

is commutative. If $w$ is archimedean, since $p$ is odd, then $\mathrm{H}^{1}\left(F_{\infty, w^{\prime}}, M_{s}\right)$ is trivial, and if $w$ is non-archimedean, then $\mathrm{H}_{\mathrm{unr}}^{1}\left(F_{\infty, w^{\prime}}, M_{s}\right)$ is trivial [26, Sect. A.2.4].

We now look at the kernel of the central vertical map in diagram (10). From the inflationrestriction exact sequence, it is $\mathrm{H}^{1}\left(F_{\infty, w^{\prime}} / F_{n, w}, M_{s}^{G_{F_{\infty}, w^{\prime}}}\right)$. If $w$ is archimedean, it splits completely in $F_{\infty} / F_{n}$ so this group is trivial. If $w$ is non-archimedean, it finitely decomposes in $F_{\infty} / F_{n}$, so that $\operatorname{Gal}\left(F_{\infty, w^{\prime}} / F_{n, w}\right) \simeq \mathbf{Z}_{p}$ and is topologically generated by an element $\gamma_{n}$. Thus $\mathrm{H}^{1}\left(F_{\infty, w^{\prime}} / F_{n, w}, M_{S}^{G_{F, w^{\prime}}}\right)$ is isomorphic to

$$
M_{S}^{G_{F}}{ }_{\infty, w^{\prime}} /\left(\gamma_{n}-1\right) M_{S}^{G_{F}}{ }_{\infty, w^{\prime}} .
$$

One has the short exact sequence

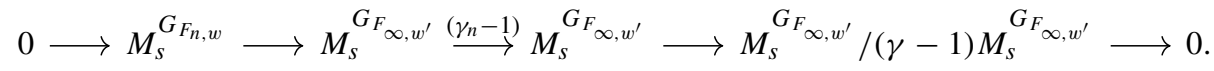

For all but finitely many $s \in \mathbf{Z}, M_{s}^{G_{F_{n, w}}}$ is finite for every $n$, hence $M_{s}^{G_{F_{\infty, w^{\prime}}}} /(\gamma-1) M_{s}^{G_{F_{\infty, w^{\prime}}}}$ is finite. So, $\left(M_{S}^{G_{F}}{ }^{\infty, w^{\prime}}\right)$ div the maximal divisible subgroup of $M_{S}^{G_{F_{\infty}, w^{\prime}}}$ is contained in $\left(\gamma_{n}-1\right) M_{S}^{G_{F_{\infty, w^{\prime}}}}$ and the order of $M_{s}^{G_{F_{\infty, w^{\prime}}}} /\left(\gamma_{n}-1\right) M_{S}^{G_{F_{\infty, w^{\prime}}}}$ is bounded by the one of $M_{s}^{G_{F}, w^{\prime}} /\left(M_{s}^{G_{F}}{ }^{G_{w} w^{\prime}}\right)_{\mathrm{div}}$.

Thus, the snake lemma applied to the diagram (10) implies that the map

$$
\frac{\mathrm{H}^{1}\left(F_{n, w}, M_{s}\right)}{\mathrm{H}_{\mathrm{unr}}^{1}\left(F_{n, w}, M_{s}\right)} \rightarrow\left(\frac{\mathrm{H}^{1}\left(F_{\infty, w^{\prime}}, M_{s}\right)}{\mathrm{H}_{\mathrm{unr}}^{1}\left(F_{\infty, w^{\prime}}, M_{s}\right)}\right)^{\Gamma_{n}}
$$

has finite kernel of bounded orders as $n$ varies.

Finally, the result follows from the snake lemma applied to the diagram (8).

Proposition 2.4 Assume that $\operatorname{Sel}_{\underline{I}^{c}}\left(M / F_{\infty}\right)$ is a cotorsion $\mathbf{Z}_{p}[[\Gamma]]-$ module. Then for all but finitely many $s \in \mathbf{Z}$, the map

$$
\mathrm{H}^{1}\left(F_{\Sigma} / F, M_{s}^{*}\right) \rightarrow \mathcal{P}_{\Sigma, \underline{I}}\left(M_{s}^{*} / F\right)
$$

is surjective, and, for all $s \in \mathbf{Z}$, the map

$$
\mathrm{H}^{1}\left(F_{\Sigma} / F_{\infty}, M_{s}^{*}\right) \rightarrow \mathcal{P}_{\Sigma, \underline{I}}\left(M_{s}^{*} / F_{\infty}\right)
$$

is surjective. 
Proof If $\operatorname{Sel}_{\underline{I}^{c}}\left(M / F_{\infty}\right)$ is a cotorsion $\mathbf{Z}_{p}[[\Gamma]]$-module, then, for all but finitely many $s \in \mathbf{Z}$, $\left(\operatorname{Sel}_{I^{c}}\left(M / F_{\infty}\right) \otimes \chi^{s}\right)^{\Gamma_{n}}=\left(\operatorname{Sel}_{\underline{I}^{c}}\left(M_{S} / F_{\infty}\right)\right)^{\Gamma_{n}}$ is finite for every $n$. Thus, by Lemma 2.3 and possibly avoiding another finite number of $s \in \mathbf{Z}, \operatorname{Sel}_{\underline{I}^{c}}\left(M_{s} / F_{n}\right)$ is finite for every $n$. For such an $s$ and any $n$, the finiteness of $\operatorname{Sel}_{I^{c}}\left(M_{s} / F_{n}\right)$ and Lemma 1.6 allow us to apply [10, Proposition 4.13] which says that the cokernel of

$$
f_{n,-s}: \mathrm{H}^{1}\left(F_{\Sigma} / F_{n}, M_{-s}^{*}\right) \rightarrow \mathcal{P}_{\Sigma, \underline{I}}\left(M_{-s}^{*} / F_{n}\right)
$$

is the Pontryagin dual of $\mathrm{H}^{0}\left(F_{n}, M_{s}\right)$. By (Tors.), $\mathrm{H}^{0}(F, M)=0$, thus $\mathrm{H}^{0}\left(F_{\infty}, M\right)=0$ as $\mathbf{Z}_{p}$ is a pro- $p$-group. Furthermore $M_{s} \simeq M$ as $\operatorname{Gal}\left(\bar{F} / F_{\infty}\right)$-modules, hence $\mathrm{H}^{0}\left(F_{\infty}, M_{s}\right)=0$ and finally $\mathrm{H}^{0}\left(F_{n}, M_{s}\right)$ is trivial for any $n$. Therefore, the map $f_{n,-s}$ is surjective for any $n$. Passing to direct limit relative to restriction maps, the surjection of the maps $f_{n,-s}$ implies the surjection of

$$
f_{\infty,-s}: \mathrm{H}^{1}\left(F_{\Sigma} / F_{\infty}, M_{-s}^{*}\right) \rightarrow \mathcal{P}_{\Sigma, \underline{I}}\left(M_{-s}^{*} / F_{\infty}\right) .
$$

Since the map $f_{\infty,-s}$ is the map

$$
f_{\infty}: \mathrm{H}^{1}\left(F_{\Sigma} / F_{\infty}, M^{*}\right) \rightarrow \mathcal{P}_{\Sigma, \underline{I}}\left(M^{*} / F_{\infty}\right)
$$

twisted by $\chi_{\mid \Gamma}^{-s}$, this concludes the proof of the proposition.

Lemma 2.5 For all $s \in \mathbf{Z}$, the restriction map

$$
\mathcal{P}_{\Sigma, \underline{I}}\left(M_{s}^{*} / F\right) \rightarrow \mathcal{P}_{\Sigma, \underline{I}}\left(M_{s}^{*} / F_{\infty}\right)^{\Gamma}
$$

is surjective.

Proof We have

$$
\mathcal{P}_{\Sigma, \underline{I}}\left(M_{s}^{*} / F_{\infty}\right)=\prod_{w \in \Sigma, w \nmid p} \frac{\mathrm{H}^{1}\left(F_{\infty, w}, M_{s}^{*}\right)}{\mathrm{H}_{\mathrm{unr}}^{1}\left(F_{\infty, w}, M_{s}^{*}\right)} \times \prod_{v \mid p} \frac{\mathrm{H}^{1}\left(F_{v, \infty}, M_{s}^{*}\right)}{\mathrm{H}_{I_{v}}^{1}\left(F_{v, \infty}, M_{s}^{*}\right)} .
$$

If $w$ is archimedean, since $p$ is odd, $\mathrm{H}^{1}\left(F_{\infty, w}, M_{s}^{*}\right)$ is trivial. If $v$ is a non-archimedean prime of $F$ not dividing $p$, the surjection

$$
\frac{\mathrm{H}^{1}\left(F_{v}, M_{s}^{*}\right)}{\mathrm{H}_{\mathrm{unr}}^{1}\left(F_{v}, M_{s}^{*}\right)} \rightarrow\left(\prod_{w \mid v} \frac{\mathrm{H}^{1}\left(F_{\infty, w}, M_{s}^{*}\right)}{\mathrm{H}_{\mathrm{unr}}^{1}\left(F_{\infty, w}, M_{s}^{*}\right)}\right)^{\Gamma}
$$

follows from the fact that $\mathrm{H}_{\mathrm{unr}}^{1}\left(F_{\infty, w}, M_{s}^{*}\right)$ is trivial and $\Gamma$ has $p$-cohomological dimension 1. Finally, if $v$ is a prime of $F$ dividing $p$, then the Pontryagin dual of $\mathrm{H}_{I_{v}}^{1}\left(F_{v, \infty}, M^{*}\right)$ is contained in a free $\mathbf{Z}_{p}[[\Gamma]]$-module by Lemma 1.2 , thus $\mathrm{H}_{I_{v}}^{1}\left(F_{v, \infty}, M^{*}\right)_{\Gamma}=0$. Hence, $\mathrm{H}_{I_{v}}^{1}\left(F_{v, \infty}, M_{s}^{*}\right)_{\Gamma}=0$ and we have an exact sequence

$$
0 \rightarrow \mathrm{H}_{I_{v}}^{1}\left(F_{v, \infty}, M_{s}^{*}\right)^{\Gamma} \rightarrow \mathrm{H}^{1}\left(F_{v, \infty}, M_{s}^{*}\right)^{\Gamma} \rightarrow\left(\frac{\mathrm{H}^{1}\left(F_{v, \infty}, M_{s}^{*}\right)}{\mathrm{H}_{I_{v}}^{1}\left(F_{v, \infty}, M_{s}^{*}\right)}\right)^{\Gamma} \rightarrow 0 .
$$

By (Tors.), we know that $\mathrm{H}^{1}\left(F_{v}, M_{s}^{*}\right) \simeq \mathrm{H}^{1}\left(F_{v, \infty}, M_{s}^{*}\right)^{\Gamma}$. Thus, by definition of $\mathrm{H}_{I_{v}}^{1}\left(F_{v}, M_{s}^{*}\right)$ and the exact sequence (11), the map

$$
\frac{\mathrm{H}^{1}\left(F_{v}, M_{s}^{*}\right)}{\mathrm{H}_{I_{v}}^{1}\left(F_{v}, M_{s}^{*}\right)} \rightarrow\left(\frac{\mathrm{H}^{1}\left(F_{v, \infty}, M_{s}^{*}\right)}{\mathrm{H}_{I_{v}}^{1}\left(F_{v, \infty}, M_{s}^{*}\right)}\right)^{\Gamma}
$$

is surjective. 
Lemma 2.6 For all $s \in \mathbf{Z}$, the $\mathbf{Z}_{p}[[\Gamma]]$-corank of $\mathcal{P}_{\Sigma, \underline{I}}\left(M_{s}^{*} / F_{\infty}\right)$ is $g_{+}$.

Proof If $w$ is archimedean, since $p$ is odd, $\mathrm{H}^{1}\left(F_{\infty, w}, M_{s}^{*}\right)$ is trivial. If $w$ is a non-archimedean prime not dividing $p$ above a prime $v$ of $F$, by [9, Proposition 2], $\mathrm{H}^{1}\left(F_{\infty, w}, M_{s}^{*}\right)$ is cotorsion. Finally, by definition, the Pontryagin dual of $\frac{\mathrm{H}^{1}\left(F_{v, \infty}, M^{*}\right)}{\mathrm{H}_{I_{v}}^{1}\left(F_{v, \infty}, M^{*}\right)}$ is isomorphic to $\operatorname{Ker} \operatorname{Col}_{T, I_{v}}$ which is of rank $g_{v}-\left|I_{v}\right|$ by Lemma 1.2. Since

$$
\frac{\mathrm{H}^{1}\left(F_{v, \infty}, M_{s}^{*}\right)}{\mathrm{H}_{I_{v}}^{1}\left(F_{v, \infty}, M_{s}^{*}\right)}=\frac{\mathrm{H}^{1}\left(F_{v, \infty}, M^{*}\right) \otimes \chi_{\mid \Gamma}^{s}}{\mathrm{H}_{I_{v}}^{1}\left(F_{v, \infty}, M^{*}\right) \otimes \chi_{\mid \Gamma}^{s}},
$$

the corank of $\frac{\mathrm{H}^{1}\left(F_{v, \infty}, M_{s}^{*}\right)}{\mathrm{H}_{I_{v}}^{1}\left(F_{v, \infty}, M_{s}^{*}\right)}$ is also $g_{v}-\left|I_{v}\right|$. Therefore, from our choice of $\underline{I}$, the corank of $\mathcal{P}_{\Sigma, \underline{I}}\left(M_{s}^{*} / F_{\infty}\right)$ is

$$
\sum_{v \mid p} g_{v}-\left|I_{v}\right|=g-g_{-}=g_{+}
$$

Proposition 2.4 and Lemma 2.6 enable to compute the corank of the Bloch-Kato Selmer group.

Corollary 2.7 Assume that $\operatorname{Sel}_{\underline{I}}\left(M^{*} / F_{\infty}\right)$ and $\operatorname{Sel}_{\underline{I}^{c}}\left(M / F_{\infty}\right)$ are cotorsion $\mathbf{Z}_{p}[[\Gamma]]$-modules. Then the $\mathbf{Z}_{p}[[\Gamma]]$-corank of $\operatorname{Sel}_{\mathrm{BK}}\left(M^{*} / F_{\infty}\right)$ is $g_{+}$.

Proof By our hypotheses, the representation $\mathcal{M}_{p}$ does not contain a non-trivial sub- $G_{F^{-}}$ representation with stricly positive Hodge-Tate weights at each prime $v$ of $F$ dividing $p$ and $\left(\mathcal{M}_{p}\right)^{G_{F v, \infty}}=0$. Hence, by [27, Corollaire 0.5], we have

$$
\mathrm{H}_{f}^{1}\left(F_{v, \infty}, M^{*}\right)=\mathrm{H}^{1}\left(F_{v, \infty}, M^{*}\right) .
$$

Thus, Eq. (12) combined with Proposition 2.4 gives the commutative diagram

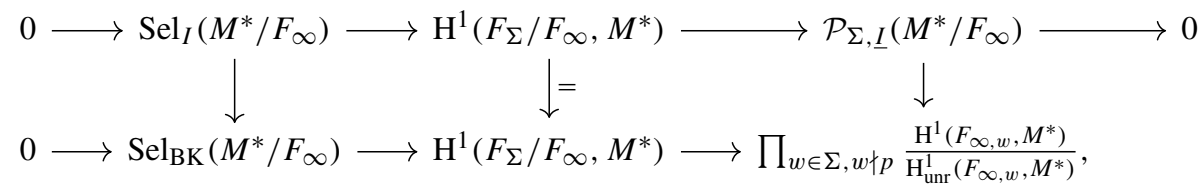

which induces, by the snake Lemma, the short exact sequence

$$
0 \rightarrow \operatorname{Sel}_{\underline{I}}\left(M^{*} / F_{\infty}\right) \rightarrow \operatorname{Sel}_{\mathrm{BK}}\left(M^{*} / F_{\infty}\right) \rightarrow \prod_{v \mid p} \frac{\mathrm{H}^{1}\left(F_{v, \infty}, M^{*}\right)}{\mathrm{H}_{I_{v}}^{1}\left(F_{v, \infty}, M^{*}\right)} \rightarrow 0
$$

The Corollary follows from the hypothesis that $\operatorname{Sel}_{\underline{I}}\left(M^{*} / F_{\infty}\right)$ is cotorsion and (the proof of) Lemma 2.6.

Proposition 2.8 Assume that $\operatorname{Sel}_{\underline{I}}\left(M^{*} / F_{\infty}\right)$ and $\operatorname{Sel}_{\underline{I}^{c}}\left(M / F_{\infty}\right)$ are cotorsion $\mathbf{Z}_{p}[[\Gamma]]-$

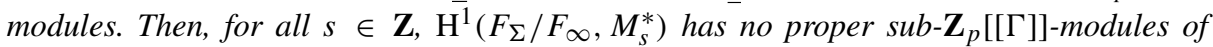
finite index. 
Proof Since $\mathrm{H}^{1}\left(F_{\Sigma} / F_{\infty}, M_{s}^{*}\right)=\mathrm{H}^{1}\left(F_{\Sigma} / F_{\infty}, M^{*}\right) \otimes \chi_{\mid \Gamma}^{s}$, it is enough to prove the proposition for $\mathrm{H}^{1}\left(F_{\Sigma} / F_{\infty}, M^{*}\right)$. By Proposition 2.4, we have the short exact sequence

$$
0 \rightarrow \operatorname{Sel}_{\underline{I}}\left(M^{*} / F_{\infty}\right) \rightarrow \mathrm{H}^{1}\left(F_{\Sigma} / F_{\infty}, M^{*}\right) \rightarrow \mathcal{P}_{\Sigma, \underline{I}}\left(M^{*} / F_{\infty}\right) \rightarrow 0 .
$$

We assume that $\operatorname{Sel}_{I}\left(M^{*} / F_{\infty}\right)$ is a cotorsion $\mathbf{Z}_{p}[[\Gamma]]$-module. The above short exact sequence then forces the $\mathbf{Z}_{p}[[\Gamma]]$-coranks of $\mathrm{H}^{1}\left(F_{\Sigma} / F_{\infty}, M^{*}\right)$ and $\mathcal{P}_{\Sigma, \underline{I}}\left(M^{*} / F_{\infty}\right)$ to be equal. Thus, by Lemma 2.6, we have

$$
\operatorname{corank}_{\mathbf{z}_{p}[[\Gamma]]} \mathrm{H}^{1}\left(F_{\Sigma} / F_{\infty}, M^{*}\right)=g_{+} .
$$

On the other hand, from the global Euler-Poincaré characteristic formula [9, Proposition 3], we have

$$
\operatorname{corank}_{\mathbf{Z}_{p}[[\Gamma]]} \mathrm{H}^{1}\left(F_{\Sigma} / F_{\infty}, M^{*}\right)=\operatorname{corank}_{\mathbf{Z}_{p}[[\Gamma]]} \mathrm{H}^{2}\left(F_{\Sigma} / F_{\infty}, M^{*}\right)+\delta\left(F, \mathcal{M}_{p}^{*}\right),
$$

with

$$
\delta\left(F, \mathcal{M}_{p}^{*}\right)=\sum_{v \text { complex }} \operatorname{dim}_{\mathbf{Q}_{p}} \mathcal{M}_{p}^{*}+\sum_{v \text { real }} \operatorname{dim}_{\mathbf{Q}_{p}}\left(\mathcal{M}_{p}^{*}\right)^{-}
$$

where $v$ runs through archimedean primes of $F$ and, for a real prime $v$, and $\operatorname{dim}_{\mathbf{Q}_{p}}\left(\mathcal{M}_{p}^{*}\right)^{-}$ is the dimension of the -1-eigenspace for a complex conjugation above $v$ acting on $\mathcal{M}_{p}$. From [9, Eq. (34)], we have

$$
\delta\left(F, \mathcal{M}_{p}^{*}\right)=\operatorname{dim}_{\mathbf{Q}_{p}}\left(\operatorname{Ind}_{F}^{\mathbf{Q}} \mathcal{M}_{p}^{*}\right)^{-}=g_{+} .
$$

Thus, $\mathrm{H}^{2}\left(F_{\Sigma} / F_{\infty}, M^{*}\right)$ is a cotorsion $\mathbf{Z}_{p}[[\Gamma]]$-module. But by [9, Proposition 4], $\mathrm{H}^{2}\left(F_{\Sigma} / F_{\infty}, M^{*}\right)$ is a cofree $\mathbf{Z}_{p}[[\Gamma]]-$ module, hence $\mathrm{H}^{2}\left(F_{\Sigma} / F_{\infty}, M^{*}\right)=0$ and the proposition follows from [9, Proposition 5].

Remark 2.9 The weak Leopoldt conjecture [26, Sect. 1.3 and Appendix B] would also imply that $\mathrm{H}^{1}\left(F_{\Sigma} / F_{\infty}, M^{*}\right)$ has no proper sub- $\mathbf{Z}_{p}[[\Gamma]]$-modules of finite index. Indeed, by the weak Leopoldt conjecture, $\mathrm{H}^{2}\left(F_{\Sigma} / F_{\infty}, M^{*}\right)$ is trivial and we can apply [9, Proposition 5].

Proof of Theorem 2.1 For any $s \in \mathbf{Z}$, since $\Gamma \simeq \mathbf{Z}_{p}$ has $p$-cohomological dimension 1, the restriction map

$$
\mathrm{H}^{1}\left(F_{\Sigma} / F, M_{s}^{*}\right) \rightarrow \mathrm{H}^{1}\left(F_{\Sigma} / F_{\infty}, M_{s}^{*}\right)^{\Gamma}
$$

is surjective. Thus, combined with Proposition 2.4 and Lemma 2.5, for all but finitely many $s \in \mathbf{Z}$, we obtain the commutative diagram

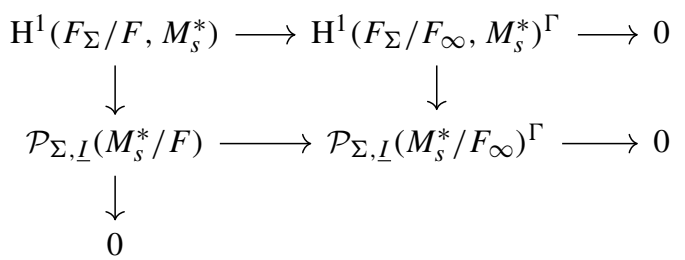

which implies that

$$
\mathrm{H}^{1}\left(F_{\Sigma} / F_{\infty}, M_{s}^{*}\right)^{\Gamma} \rightarrow \mathcal{P}_{\Sigma, \underline{I}}\left(M_{s}^{*} / F_{\infty}\right)^{\Gamma}
$$

is surjective. 
By Proposition 2.4, we have the short exact sequence

$$
0 \rightarrow \operatorname{Sel}_{\underline{I}}\left(M_{s}^{*} / F_{\infty}\right) \rightarrow \mathrm{H}^{1}\left(F_{\Sigma} / F_{\infty}, M_{s}^{*}\right) \rightarrow \mathcal{P}_{\Sigma, \underline{I}}\left(M_{s}^{*} / F_{\infty}\right) \rightarrow 0 .
$$

Taking $\Gamma$-invariants gives the long exact sequence

$$
\mathrm{H}^{1}\left(F_{\Sigma} / F_{\infty}, M_{s}^{*}\right)^{\Gamma} \rightarrow \mathcal{P}_{\Sigma, \underline{I}}\left(M_{s}^{*} / F_{\infty}\right)^{\Gamma} \rightarrow \operatorname{Sel}_{\underline{I}}\left(M_{s}^{*} / F_{\infty}\right)_{\Gamma} \rightarrow \mathrm{H}^{1}\left(F_{\Sigma} / F_{\infty}, M_{s}^{*}\right)_{\Gamma} .
$$

Since the first map is surjective, $\operatorname{Sel}_{\underline{I}}\left(M_{s}^{*} / F_{\infty}\right)_{\Gamma} \rightarrow \mathrm{H}^{1}\left(F_{\Sigma} / F_{\infty}, M_{s}^{*}\right)_{\Gamma}$ is injective. Furthermore, $\mathrm{H}^{1}\left(F_{\Sigma} / F_{\infty}, M_{s}^{*}\right)_{\Gamma}$ is trivial by Proposition 2.8. Thus, $\operatorname{Sel}_{\underline{I}}\left(M_{s}^{*} / F_{\infty}\right)_{\Gamma}=0$ which implies the result.

\subsection{An application: computation of the Euler-Poincaré characteristic}

We now choose a strongly admissible basis as in Lemma 1.11 and any $\underline{I} \in \mathcal{I}_{p}$.

For $v$ a non-archimedean prime not dividing $p$, we recall the definition of the Tamagawa number of $T$ at $v$ [8, I Sect. 4].

If $N$ is $\mathbf{Q}_{p}$-vector space of finite dimension $d$ (respectively a free $\mathbf{Z}_{p}$-module of rank $d$ ), we denote by $N^{-1}$ its linear dual and we set $\operatorname{det}_{\mathbf{Q}_{p}} N=\wedge_{\mathbf{Q}_{p}}^{d} N\left(\operatorname{respectively~} \operatorname{det}_{\mathbf{z}_{p}} N=\wedge_{\mathbf{Z}_{p}}^{d} N\right)$. If $N$ is now a finitely generated $\mathbf{Z}_{p}$-module, we define the determinant of $N$ over $\mathbf{Z}_{p}$ as

$$
\operatorname{det}_{\mathbf{z}_{p}} N=\left(\operatorname{det}_{\mathbf{z}_{p}} N_{-1}\right)^{-1} \otimes \operatorname{det}_{\mathbf{z}_{p}} N_{0},
$$

where

$$
0 \rightarrow N_{-1} \rightarrow N_{0} \rightarrow N \rightarrow 0
$$

is a resolution of $N$ by free $\mathbf{Z}_{p}$-modules of finite ranks $N_{-1}$ and $N_{0}$.

We recall that we denote by $\mathcal{M}_{p}$ the $p$-adic realization of our fixed motive $\mathcal{M}$. Let Frob $_{v}$ be the geometric Frobenius in $\operatorname{Gal}\left(F_{v \text {,unr }} / F_{v}\right)$, we have an exact sequence of $\mathbf{Q}_{p}$-vector spaces

$$
0 \rightarrow \mathrm{H}^{0}\left(F_{v}, \mathcal{M}_{p}\right) \rightarrow \mathrm{H}^{0}\left(F_{v, \text { unr }}, \mathcal{M}_{p}\right) \stackrel{1-\mathrm{Frob}_{v}}{\longrightarrow} \mathrm{H}^{0}\left(F_{v, \mathrm{unr}}, \mathcal{M}_{p}\right) \rightarrow \mathrm{H}_{\mathrm{unr}}^{1}\left(F_{v}, \mathcal{M}_{p}\right) \rightarrow 0
$$

which induces an isomorphism of $\mathbf{Q}_{p}$-vector spaces

$$
\iota_{\mathcal{M}_{p}, v}: \operatorname{det}_{\mathbf{Q}_{p}} \mathrm{H}^{0}\left(F_{v}, \mathcal{M}_{p}\right) \otimes\left(\operatorname{det}_{\mathbf{Q}_{p}} \mathrm{H}_{\mathrm{unr}}^{1}\left(F_{v}, \mathcal{M}_{p}\right)\right)^{-1} \rightarrow \mathbf{Q}_{p} .
$$

Then

$$
\operatorname{det}_{\mathbf{z}_{p}} \mathrm{H}^{0}\left(F_{v}, T\right) \otimes\left(\operatorname{det}_{\mathbf{z}_{p}} \mathrm{H}_{\text {unr }}^{1}\left(F_{v}, T\right)\right)^{-1},
$$

is a $\mathbf{Z}_{p}$-lattice inside $\operatorname{det}_{\mathbf{Q}_{p}} \mathrm{H}^{0}\left(F_{v}, \mathcal{M}_{p}\right) \otimes\left(\operatorname{det}_{\mathbf{Q}_{p}} \mathrm{H}_{\text {unr }}^{1}\left(F_{v}, \mathcal{M}_{p}\right)\right)^{-1}$ and the Tamagawa number of $T$ at $v$, denoted by $\operatorname{Tam}_{v}(T)$, is defined as the unique power of $p$ such that

$$
\iota_{\mathcal{M}}, v\left(\operatorname{det}_{\mathbf{z}_{p}} \mathrm{H}^{0}\left(F_{v}, T\right) \otimes\left(\operatorname{det}_{\mathbf{z}_{p}} \mathrm{H}_{\text {unr }}^{1}\left(F_{v}, T\right)\right)^{-1}\right)=\mathbf{Z}_{p} \cdot \operatorname{Tam}_{v}(T) .
$$

We can now deduce the following corollary on the leading term of the algebraic $p$-adic $L$-function, which is a generalization of Kim's result on Kobayashi's plus/minus Selmer groups [16, Theorem 1.2].

Corollary 2.10 Assume that $\operatorname{Sel}_{\mathrm{BK}}\left(M^{*} / F\right)$ is finite, and that $\operatorname{Sel}_{I^{c}}\left(M / F_{\infty}\right)$ is a cotorsion $\mathbf{Z}_{p}[[\Gamma]]$-module. Denote by $\left(f_{\underline{I}}\right) \subset \mathbf{Z}_{p}[[X]]$ the characteristic ideal of $\operatorname{Sel}_{\underline{I}}\left(M^{*} / F_{\infty}\right)$. Then, up to a unit,

$$
f_{\underline{I}}(0)=\left|\operatorname{Sel}_{\mathrm{BK}}\left(M^{*} / F\right)\right| \cdot \prod_{v \nmid p} \operatorname{Tam}_{v}(T) .
$$


Proof First, we remark that the hypothesis implies that $\operatorname{Sel}_{\underline{I}}\left(M^{*} / F_{\infty}\right)$ is a cotorsion $\mathbf{Z}_{p}[[\Gamma]]$ module since, by Lemma $1.11, \operatorname{Sel}_{\mathrm{BK}}\left(M^{*} / F\right)=\operatorname{Sel}_{I}\left(M^{*} / F\right)$, and we have a "control theorem" (Lemma 2.3). Thus $\operatorname{Sel}_{\underline{I}}\left(M^{*} / F_{\infty}\right)^{\Gamma}$ is finite, which implies that $\operatorname{Sel}_{\underline{I}}\left(M^{*} / F_{\infty}\right)$ is cotorsion.

Up to a unit, we have

$$
\begin{aligned}
f_{\underline{I}}(0) & =\left|\operatorname{Sel}_{\underline{I}}\left(M^{*} / F_{\infty}\right)^{\Gamma}\right| /\left|\operatorname{Sel}_{\underline{I}}\left(M^{*} / F_{\infty}\right)_{\Gamma}\right| \\
& =\left|\operatorname{Sel}_{\underline{I}}\left(M^{*} / F_{\infty}\right)^{\Gamma}\right|,
\end{aligned}
$$

where the first relation is [10, Lemma 4.2] and the second is Theorem 2.1.

It remains to relate the right hand side of the formula (13) to $\left|\operatorname{Sel}_{I}\left(M^{*} / F_{\infty}\right)^{\Gamma}\right|$. It is done by studying the commutative diagram

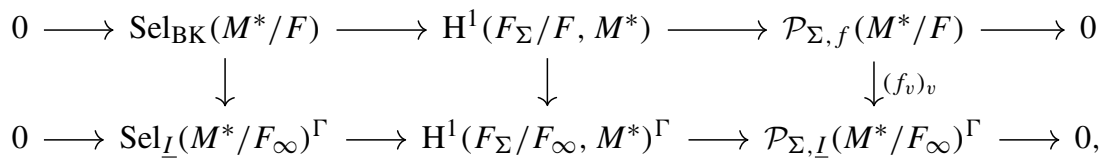

where the surjection at the end of the top row is Proposition 2.4 and the one at the bottom row is due to Theorem 2.1. As we mentioned in the proof of Lemma 2.3, by (Tors.), the central map is an isomorphism by the inflation-restriction exact sequence. Hence, by the snake lemma, we have

$$
\left|\operatorname{Sel}_{\underline{I}}\left(M^{*} / F_{\infty}\right)^{\Gamma}\right|=\left|\operatorname{Sel}_{\mathrm{BK}}\left(M^{*} / F\right)\right| . \prod_{v \in \Sigma}\left|\operatorname{Ker} f_{v}\right| .
$$

We now compute $\left|\operatorname{Ker} f_{v}\right|$. As we have already remarked, the archimedean part is trivial since $p$ is odd, and if $v$ divides $p$, then $f_{v}$ is injective (see the proof of Lemma 2.3). Finally, if $v$ is a non-archimedean prime not dividing $p$, then $\operatorname{Ker} f_{v}$ is the orthogonal complement under Tate's local pairing of the projection

$$
\operatorname{Im}\left(\mathrm{H}_{\mathrm{Iw}}^{1}\left(F_{v}, T\right) \stackrel{f_{v}^{*}}{\longrightarrow} \mathrm{H}_{\mathrm{unr}}^{1}\left(F_{v}, T\right)\right) .
$$

By [24, Lemme 2.2.5], we have $\left|\operatorname{Coker} f_{v}^{*}\right|=\operatorname{Tam}_{v}(T)$. Thus, $\left|\operatorname{Ker} f_{v}\right|=\operatorname{Tam}_{v}(T)$. Since $\operatorname{Tam}_{v}(T)=1$ at primes $v$ where $M^{*}$ is unramified and all the ramified primes of $M^{*}$ are contained in $\Sigma$, we can extend the product in (15) over all nonarchimedean primes not dividing $p$. The corollary follows from (14) combined with (15).

\section{Congruences}

Let $\mathcal{M}^{\prime}$ be another motive and $T^{\prime}$ a $G_{F}$-stable $\mathbf{Z}_{p}$-lattice inside $\mathcal{M}_{p}^{\prime}$ the $p$-adic realization of $\mathcal{M}^{\prime}$ satisfying all the hypotheses (H.-T.), (Cryst.), (Tors.), (Fil.) and (Slopes). We shall simply add a superscript $(\cdot)^{\prime}$ to the various object associated to $T$ to denote the similar object associated to $T^{\prime}$ (e.g. $M^{\prime}=T^{\prime} \otimes \mathbf{Q}_{p} / \mathbf{Z}_{p}$ ).

From now on, we assume that

$$
T / p T \simeq T^{\prime} / p T^{\prime},
$$

(Cong.)

as $G_{F}$-representations. The goal of this section is to compare the signed Selmer groups of $M^{*}$ and $M^{\prime}$,* under the hypothesis (Cong.). We begin by studying the implication of this congruence on the signed Coleman maps. 


\subsection{Wach modules}

We succinctly recall what we need for our purpose and refer the reader to [1,2] for details. Let $v$ be a prime of $F$ dividing $p$ and let $\mathbf{A}_{F_{v}}^{+}$be the ring $\mathcal{O}_{F_{v}}[[\pi]]$ equipped with the semilinear action by the Frobenius $\phi$ which acts as the absolute Frobenius on $\mathcal{O}_{F_{v}}$ and on $\pi$ by

$$
\phi(\pi)=(\pi+1)^{p}-1,
$$

and with an action of $\operatorname{Gal}\left(F_{v}\left(\mu_{p^{\infty}}\right) / F_{v}\right)$ given by

$$
g(\pi)=(\pi+1)^{\chi(g)}-1, \forall g \in \operatorname{Gal}\left(F_{v}\left(\mu_{p^{\infty}}\right) / F_{v}\right) .
$$

There exists a Wach module $\mathbf{N}_{v}(T)$ attached to $\left.T\right|_{G_{F_{v}}}$ (and similarly to $\left.T^{\prime}\right|_{G_{F_{v}}}$ ), which is a free $\mathbf{A}_{F_{v}}^{+}$-module of rank $\operatorname{dim}_{\mathbf{Q}_{p}}\left(\mathcal{M}_{p}\right)$ equipped with an action of $\operatorname{Gal}\left(F_{v}\left(\mu_{p^{\infty}}\right) / F_{v}\right)$ and a $\phi$-linear endomorphism of $\mathbf{N}_{v}(T)\left[\frac{1}{\pi}\right]$, which we still denote by $\phi$, commuting with the Galois action. We denote by $\phi^{*} \mathbf{N}_{v}(T)$ the $\mathbf{A}_{F_{v}}^{+}$-module generated by $\phi\left(\mathbf{N}_{v}(T)\right)$.

The Dieudonné module associated to $T$ is defined via the Wach module by

$$
\mathbf{N}_{v}(T) / \pi \mathbf{N}_{v}(T)=\mathbf{D}_{\text {cris }, v}(T),
$$

where the filtration on $\mathbf{N}_{v}(T)$ inducing the one on $\mathbf{D}_{\text {cris }, v}(T)$ is

$$
\operatorname{Fil}^{i} \mathbf{N}_{v}(T)=\left\{x \in \mathbf{N}_{v}(T), \phi(x) \in(\phi(\pi) / \pi)^{i} \mathbf{N}_{v}(T)\right\},
$$

One also recovers the first Iwasawa cohomology group from the Wach module by an isomorphism of $\mathbf{Z}_{p}\left[\left[\operatorname{Gal}\left(F_{v}\left(\mu_{p^{\infty}}\right) / F_{v}\right)\right]\right]$-modules

$$
h_{T}^{1}: \mathrm{H}_{\mathrm{Iw}}^{1}\left(F_{v}, T\right) \stackrel{\sim}{\rightarrow} \mathbf{N}_{v}(T)^{\psi=1},
$$

where $\psi$ is a left inverse for $\phi$.

We compare the Wach module of $T$ and $T^{\prime}$ modulo $p$. Since the Hodge-Tate weights of $T$ and $T^{\prime}$ are in [0,1], the following theorem is a special case of [2, Théorème IV.1.1].

Theorem 3.1 The isomorphism (Cong.) induces an isomorphism of $\mathbf{A}_{F_{v}}^{+}$-modules

$$
\mathbf{N}_{v}(T) / p \mathbf{N}_{v}(T) \simeq \mathbf{N}_{v}\left(T^{\prime}\right) / p \mathbf{N}_{v}\left(T^{\prime}\right),
$$

which is compatible with the filtration, the Galois action and the action of $\phi$.

\subsection{Congruences of signed Coleman maps}

We now follow the construction of the signed Coleman maps as given in [5, Sect. 2] keeping track of the congruences modulo $p$.

First, note that by (17) and Theorem 3.1, we have a $\mathbf{Z}_{p}\left[\left[\operatorname{Gal}\left(F_{v}\left(\mu_{p^{\infty}}\right) / F_{v}\right)\right]\right]$-isomorphism

$$
\mathrm{H}_{\mathrm{Iw}}^{1}\left(F_{v}, T\right) / p \simeq \mathrm{H}_{\mathrm{Iw}}^{1}\left(F_{v}, T^{\prime}\right) / p .
$$

Also, combining (16) and Theorem 3.1, the Dieudonné modules associated to $T$ and $T^{\prime}$ are isomorphic modulo $p$. We fix good bases for $\mathbf{D}_{\text {cris }, v}(T)$ and $\mathbf{D}_{\text {cris }, v}\left(T^{\prime}\right)$ compatible with the isomorphism given in Theorem 3.1 in the sense that they have the same image under (16).

Lemma 3.2 For $n \geqslant 1$, there exists a unique $\Lambda$-homomorphism

$$
\mathcal{L}_{T}^{(n)}: \mathrm{H}_{\mathrm{IW}}^{1}\left(F_{v}, T\right) \rightarrow \Lambda_{n} \otimes \mathbf{z}_{p} \mathbf{D}_{\text {cris }, v}(T)
$$


such that

$$
\phi^{-n-1} \circ \mathcal{L}_{T} \equiv \mathcal{L}_{T}^{(n)} \bmod \omega_{n} .
$$

Furthermore, the applications $\mathcal{L}_{T}^{(n)}$ and $\mathcal{L}_{T^{\prime}}^{(n)}$ are congruent modulo p, i.e. the diagram

$$
\begin{gathered}
\mathrm{H}_{\mathrm{Iw}}^{1}\left(F_{v}, T\right) / p^{p^{(n)}} \stackrel{\bmod p}{\longrightarrow} \Lambda_{n} \otimes \mathbf{z}_{p} \mathbf{D}_{\text {cris }, v}(T) / p \\
\downarrow \simeq \quad \mathcal{L}^{(n)} \bmod p \\
\mathrm{H}_{\mathrm{IW}}^{1}\left(F_{v}, T^{\prime}\right) / p^{T^{\prime}} \longrightarrow \Lambda_{n} \otimes_{\mathbf{z}_{p}} \mathbf{D}_{\text {cris }, v}\left(T^{\prime}\right) / p
\end{gathered}
$$

is commutative.

Proof The first statement is Proposition 2.9 of op. cit.. We follow its proof to prove the second. Perrin-Riou's big logarithm $\mathcal{L}_{T}$ is given by

$$
(\mathfrak{M} \otimes 1)^{-1} \circ(1-\phi) \circ\left(h_{T}\right)^{-1}
$$

where $\mathfrak{M}$ is the Mellin transform which maps elements of $\mathcal{H}$ to overconvergent power series in $\pi$, whose set we denote $\mathbf{B}_{\text {rig, } F_{v}}^{+}$. The Mellin transform preserves integrality and the ideal $\left(\omega_{n}\right)$ corresponds to $\left(\phi^{n+1}(\pi)\right)$.

The first statement then follows from a study (Lemma 3.44 of op. cit.) of the map

$$
\phi^{-n-1} \circ(1-\phi): \mathbf{N}_{v}(T)^{\psi=1} \rightarrow\left(\phi^{*} \mathbf{N}_{v}(T)\right)^{\psi=0} \hookrightarrow \mathbf{B}_{\mathrm{rig}, F_{v}}^{+} \otimes \mathbf{z}_{p} \mathbf{D}_{\text {cris }, v}(T)
$$

which shows that, for $x \in \mathbf{N}_{v}(T)^{\psi=1}$, the element $\phi^{-n-1} \circ(1-\phi)(x)$ is congruent to an element of $\left(\mathbf{A}_{F_{v}}^{+}\right)^{\psi=0} \otimes \mathbf{z}_{p} \mathbf{D}_{\text {cris }, v}(T)$ modulo $\phi^{n+1}(\pi) \mathbf{B}_{\text {rig, } F_{v}}^{+} \otimes \mathbf{z}_{p} \mathbf{D}_{\text {cris }, v}(T)$. But by Theorem 3.1, the maps (19) for $T$ and $T^{\prime}$ agree modulo $p$ and we are done.

For $i \in\left\{1, \ldots, g_{v}\right\}$, we write $\mathcal{L}_{T, i}^{(n)}$ for the composition of $\mathcal{L}_{T}^{(n)}$ with the projection on the $i$-th component of the fixed basis of $\mathbf{D}_{\text {cris, } v}(T)$. We set $h_{n}$ (respectively $h_{n}^{\prime}$ ) the $\Lambda_{n^{-}}$ endomorphisms on $\oplus_{k=1}^{g_{v}} \Lambda_{n}$ given by the left multiplication by the product $C_{v, n} \cdots C_{v, 1}$ (respectively $C_{v, n}^{\prime} \cdots C_{v, 1}^{\prime}$ ).

Lemma 3.3 For $n \geqslant 1$, there exists a unique $\Lambda$-homomorphism

$$
\mathrm{Col}_{T}^{(n)}: \mathrm{H}_{\mathrm{IW}}^{1}\left(F_{v}, T\right) \rightarrow \bigoplus_{k=1}^{g_{v}} \Lambda_{n}
$$

such that

$$
\left(\begin{array}{c}
\mathcal{L}_{T, 1}^{(n)} \\
\vdots \\
\mathcal{L}_{T, g_{v}}^{(n)}
\end{array}\right) \equiv C_{v, n} \cdots C_{v, 1} \cdot \operatorname{Col}_{T}^{(n)} \quad \bmod \operatorname{Ker} h_{n}
$$

Furthermore, we have

$$
\mathrm{Col}_{T}^{(n)} \equiv \mathrm{Col}_{T^{\prime}}^{(n)} \quad \bmod p .
$$

Proof The first part is Proposition 2.10 of op. cit.. Again by Theorem 3.1 and (16), the matrices $C_{v, n}$ and $C_{v, n}^{\prime}$ are congruent modulo $p$ for all $n$. Thus, by the first part of the Lemma and Lemma 3.2, we have

$$
C_{v, n} \cdots C_{v, 1} \cdot \mathrm{Col}_{T}^{(n)} \equiv C_{v, n} \cdots C_{v, 1} \cdot \mathrm{Col}_{T^{\prime}}^{(n)} \quad \bmod \left(\operatorname{Ker} h_{n}, p\right) .
$$


Since

$$
C_{v, n}=\left(\begin{array}{c|c}
I_{d_{v}} & 0 \\
\hline 0 & \Phi_{p^{n}}(1+X) I_{g_{v}-d_{v}}
\end{array}\right) C_{v}^{-1}
$$

with $C_{v} \in \mathrm{GL}_{g_{v}}\left(\mathbf{Z}_{p}\right)$ and $\Phi_{p^{n}}(1+X)$ and $p$ are coprime, the second part follows.

By [5, Lemma 2.11 and Theorem 2.13], the maps $\left(\mathrm{Col}_{T}^{(n)}\right)_{n \geqslant 1}$ are compatible with the natural projection $\oplus_{k=1}^{g_{v}} \Lambda_{n+1} / \operatorname{Ker} h_{n+1} \rightarrow \oplus_{k=1}^{g_{v}} \Lambda_{n} / \operatorname{Ker} h_{n}$ and thus define a map to $\lim _{n} \oplus_{k=1}^{g_{v}} \Lambda_{n} / \operatorname{Ker} h_{n}$ which naturally identifies with $\oplus_{k=1}^{g_{v}} \Lambda$. By definition, this map is $\overleftarrow{\mathrm{Col}}_{T}$. Thus, by Lemma 3.3 we have:

Proposition 3.4 The Coleman maps associated to $T$ and $T^{\prime}$ are congruent modulo $p$. More precisely, if $z \in \mathrm{H}_{\mathrm{IW}}^{1}\left(F_{v}, T\right)$ and $z^{\prime} \in \mathrm{H}_{\mathrm{IW}}^{1}\left(F_{v}, T^{\prime}\right)$ have the same image under the isomorphism given in (18), then

$$
\operatorname{Col}_{T}(z) \equiv \operatorname{Col}_{T^{\prime}}\left(z^{\prime}\right) \quad \bmod p \oplus_{k=1}^{g_{v}} \Lambda .
$$

\subsection{Non-primitive signed Selmer groups}

We now define and compare the non-primitive signed Selmer groups under the hypothesis (Cong.) and deduce our main result.

The next lemma is well-known [23, Lemma 3.5.3].

Lemma 3.5 The exact sequence

$$
0 \rightarrow M^{*}[p] \rightarrow M^{*} \stackrel{p}{\rightarrow} M^{*} \rightarrow 0
$$

of $G_{F_{\infty}}$-modules induces isomorphisms

$$
\mathrm{H}^{1}\left(F_{\Sigma} / F_{\infty}, M^{*}[p]\right) \simeq \mathrm{H}^{1}\left(F_{\Sigma} / F_{\infty}, M^{*}\right)[p],
$$

and

$$
\mathrm{H}^{1}\left(F_{v, \infty}, M^{*}[p]\right) \simeq \mathrm{H}^{1}\left(F_{v, \infty}, M^{*}\right)[p],
$$

for any prime $v$ dividing $p$, and, for any non-archimedean prime $v$ not dividing $p$ or a prime of ramification of $M^{*}$,

$$
\mathrm{H}^{1}\left(F_{v, \mathrm{unr}}, M^{*}[p]\right) \simeq \mathrm{H}^{1}\left(F_{v, \mathrm{unr}}, M^{*}\right)[p] .
$$

Proof We have the exact sequence

$$
0 \rightarrow \mathrm{H}^{0}\left(F_{\infty}, M^{*}\right) / p \rightarrow \mathrm{H}^{1}\left(F_{\Sigma} / F_{\infty}, M^{*}[p]\right) \rightarrow \mathrm{H}^{1}\left(F_{\Sigma} / F_{\infty}, M^{*}\right)[p] \rightarrow 0 .
$$

Since $\mathrm{H}^{0}\left(F_{\infty}, M^{*}\right)$ is trivial by our hypothesis (Tors.), we get the first isomorphism. The same proof applies for the second isomorphism at $v$ dividing $p$. Let $v$ as in the third statement, then $v$ is not a prime of ramification for $M^{*}$, thus $\mathrm{H}^{0}\left(F_{v, \text { unr }}, M^{*}\right)=M^{*}$. Hence, we have the exact sequence

$$
0 \rightarrow M^{*} / p \rightarrow \mathrm{H}^{1}\left(F_{v, \text { unr }}, M^{*}[p]\right) \rightarrow \mathrm{H}^{1}\left(F_{v, \text { unr }}, M^{*}\right)[p] \rightarrow 0 .
$$

Since $M^{*}$ is divisible, we deduce the second isomorphism. 
Definition 3.6 Let $\Sigma_{0} \subset \Sigma$ be a subset that contains all the primes of ramification of $M^{*}$ but none of the primes of $F$ dividing $p$ nor the archimedean primes. We define the $\Sigma_{0}$-nonprimitive $\underline{I}$-Selmer groups of $M^{*}$ over $F_{\infty}$ by

$$
\operatorname{Sel}_{\underline{I}}^{\Sigma_{0}}\left(M^{*} / F_{\infty}\right)=\operatorname{Ker}\left(\mathrm{H}^{1}\left(F_{\Sigma} / F_{\infty}, M^{*}\right) \rightarrow \mathcal{P}_{\Sigma \backslash \Sigma_{0}, \underline{I}}\left(M^{*} / F_{\infty}\right)\right) .
$$

If $v$ is a prime dividing $p$, by Lemma 3.5 we have $\mathrm{H}^{1}\left(F_{v, \infty}, M^{*}[p]\right) \simeq \mathrm{H}^{1}\left(F_{v, \infty}, M^{*}\right)[p]$ and we set

$$
\mathrm{H}_{I_{v}}^{1}\left(F_{v, \infty}, M^{*}[p]\right)=\mathrm{H}_{I_{v}}^{1}\left(F_{v, \infty}, M^{*}\right)[p] \subset \mathrm{H}^{1}\left(F_{v, \infty}, M^{*}[p]\right) .
$$

We set

$$
\mathcal{P}_{\Sigma \backslash \Sigma_{0}, \underline{I}}\left(M^{*}[p] / F_{\infty}\right)=\prod_{w \in \Sigma \backslash \Sigma_{0}, w \nmid p} \mathrm{H}^{1}\left(F_{w, \mathrm{unr}}, M^{*}[p]\right) \times \prod_{w \mid p} \frac{\mathrm{H}^{1}\left(F_{w, \infty}, M^{*}[p]\right)}{\mathrm{H}_{I_{v}}^{1}\left(F_{w, \infty}, M^{*}[p]\right)} .
$$

We define the $\Sigma_{0}$-non-primitive $\underline{I}$-Selmer groups of $M^{*}[p]$ over $F_{\infty}$ by

$$
\operatorname{Sel}_{\underline{I}}^{\Sigma_{0}}\left(M^{*}[p] / F_{\infty}\right)=\operatorname{Ker}\left(\mathrm{H}^{1}\left(F_{\Sigma} / F_{\infty}, M^{*}[p]\right) \rightarrow \mathcal{P}_{\Sigma \backslash \Sigma_{0}, \underline{I}}\left(M^{*}[p] / F_{\infty}\right)\right) .
$$

Remark 3.7 By Tate's pairing (6), the Pontryagin dual of $\mathrm{H}_{I_{v}}^{1}\left(F_{v, \infty}, M^{*}[p]\right)$ is $\left(\operatorname{Im~} \operatorname{Col}_{T, I_{v}}\right) / p$.

From now on, we write abusively the $\mu$ and $\lambda$-invariants of the various Selmer groups to refer to the $\mu$ and $\lambda$-invariants of their Pontryagin duals.

Proposition 3.8 For any $\Sigma_{0} \subset \Sigma$ as in Definition 3.6, we have an isomorphism of $\mathbf{Z}_{p}[[\Gamma]]$ modules

$$
\operatorname{Sel}_{\underline{I}}^{\Sigma_{0}}\left(M^{*}[p] / F_{\infty}\right) \simeq \operatorname{Sel}_{\underline{I}}^{\Sigma_{0}}\left(M^{*} / F_{\infty}\right)[p] .
$$

Proof By Lemma 3.5, we have

$$
\mathrm{H}^{1}\left(F_{\Sigma} / F_{\infty}, M^{*}[p]\right) \simeq \mathrm{H}^{1}\left(F_{\Sigma} / F_{\infty}, M^{*}\right)[p] .
$$

Therefore, in order to prove the Proposition, it is enough to compare the local conditions defining the two Selmer groups. At $v \in \Sigma \backslash \Sigma_{0}$ and $v$ not dividing $p$, the second part of Lemma 3.5 shows that the local conditions are equivalent. Since $p$ is odd, the archimedean part is trivial. At $v$ dividing $p$, by definition the local conditions are the same.

We now relate the $\Sigma_{0}$-non-primitive signed Selmer groups to the signed Selmer groups.

Proposition 3.9 Assume that $\operatorname{Sel}_{\underline{I}^{c}}\left(M / F_{\infty}\right)$ is a cotorsion $\mathbf{Z}_{p}[[\Gamma]]-$ module. Then

$$
\operatorname{Sel}_{\underline{I}}^{\Sigma_{0}}\left(M^{*} / F_{\infty}\right) / \operatorname{Sel}_{\underline{I}}\left(M^{*} / F_{\infty}\right) \simeq \prod_{w \in \Sigma_{0}} \frac{\mathrm{H}^{1}\left(F_{\infty, w}, M^{*}\right)}{\mathrm{H}_{\mathrm{unr}}^{1}\left(F_{\infty, w}, M^{*}\right)} .
$$

Proof By Proposition 2.4, we have the commutative diagram

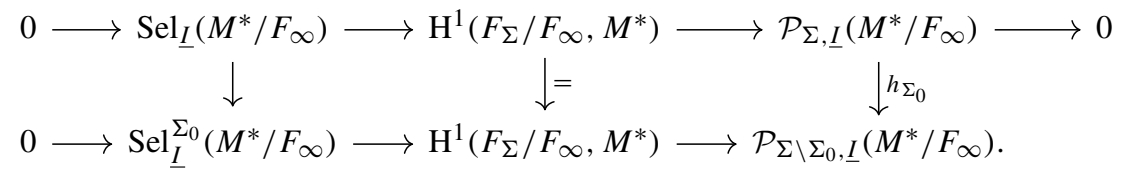


The Snake lemma applied to diagram (20) gives

$$
\operatorname{Sel}_{\underline{I}}^{\Sigma_{0}}\left(M^{*} / F_{\infty}\right) / \operatorname{Sel}_{\underline{I}}\left(M^{*} / F_{\infty}\right) \simeq \operatorname{Ker}\left(h_{\Sigma_{0}}\right)=\prod_{w \in \Sigma_{0}} \frac{\mathrm{H}^{1}\left(F_{\infty, w}, M^{*}\right)}{\mathrm{H}_{\mathrm{unr}}^{1}\left(F_{\infty, w}, M^{*}\right)} .
$$

Corollary 3.10 Assume that $\operatorname{Sel}_{\underline{I}^{c}}\left(M / F_{\infty}\right)$ and $\operatorname{Sel}_{\underline{I}}\left(M^{*} / F_{\infty}\right)$ are cotorsion $\mathbf{Z}_{p}[[\Gamma]]-$ modules. Then $\operatorname{Sel}_{I}^{\Sigma_{0}}\left(M^{*} / F_{\infty}\right)$ is a cotorsion $\mathbf{Z}_{p}[[\Gamma]]$-module. Furthermore, we have

$$
\mu\left(\operatorname{Sel}_{\underline{I}}^{\Sigma_{0}}\left(M^{*} / F_{\infty}\right)\right)=\mu\left(\operatorname{Sel}_{\underline{I}}\left(M^{*} / F_{\infty}\right)\right),
$$

and

$$
\operatorname{corank}_{\mathbf{Z}_{p}} \operatorname{Sel}_{\underline{I}}^{\Sigma_{0}}\left(M^{*} / F_{\infty}\right)=\operatorname{corank}_{\mathbf{Z}_{p}} \operatorname{Sel}_{\underline{I}}\left(M^{*} / F_{\infty}\right)+\sum_{w \in \Sigma_{0}} \operatorname{corank}_{\mathbf{Z}_{p}} \mathrm{H}^{1}\left(F_{\infty, w}, M^{*}\right) .
$$

Proof We have already noted that $\mathrm{H}_{\mathrm{unr}}^{1}\left(F_{\infty, w}, M^{*}\right)$ is trivial (by [26, Sect. A.2.4]) and that $\mathrm{H}^{1}\left(F_{\infty, w}, M^{*}\right)$ is a cotorsion $\mathbf{Z}_{p}[[\Gamma]]$-module for all $w \in \Sigma_{0}$ (by [9, Proposition 2]). Furthermore, the $\mu$-invariant of the Pontryagin dual of $\mathrm{H}^{1}\left(F_{\infty, w}, M^{*}\right)$ is zero [11, Proposition 2.4]. Thus the corollary follows from Proposition 3.9.

We have an analogue of Theorem 2.1 for $\Sigma_{0}$-non-primitive signed Selmer groups.

Proposition 3.11 Assume that $\operatorname{Sel}_{\underline{I}}\left(M^{*} / F_{\infty}\right)$ and $\operatorname{Sel}_{\underline{I}^{c}}\left(M / F_{\infty}\right)$ are cotorsion $\mathbf{Z}_{p}[[\Gamma]]-$

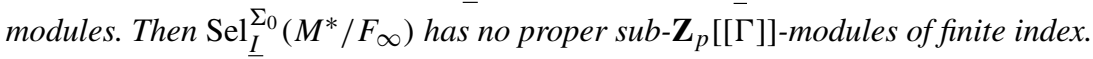

Proof From the definition of the $\Sigma_{0}$-non-primitive signed Selmer groups and Proposition 2.4, we have an analogue of Proposition 2.4 for the $\Sigma_{0}$-non-primitive signed Selmer groups. That is, in diagram (20), the surjectivity of $h_{\Sigma_{0}}$ implies that the sequence

$$
0 \rightarrow \operatorname{Sel}_{\underline{I}}^{\Sigma_{0}}\left(M^{*} / F_{\infty}\right) \rightarrow \mathrm{H}^{1}\left(F_{\Sigma} / F_{\infty}, M^{*}\right) \rightarrow \mathcal{P}_{\Sigma \backslash \Sigma_{0}, \underline{I}}\left(M^{*} / F_{\infty}\right) \rightarrow 0
$$

is exact. Similarly, by Proposition 2.4, we have

$$
0 \rightarrow \operatorname{Sel}_{\underline{I}}^{\Sigma_{0}}\left(M^{*} / F\right) \rightarrow \mathrm{H}^{1}\left(F_{\Sigma} / F, M^{*}\right) \rightarrow \mathcal{P}_{\Sigma \backslash \Sigma_{0}, \underline{I}}\left(M^{*} / F\right) \rightarrow 0 .
$$

The proof of the Proposition then follows precisely the one of Theorem 2.1, hence, we skip it.

Corollary 3.12 Assume that $\operatorname{Sel}_{\underline{I}}\left(M^{*} / F_{\infty}\right)$ and $\operatorname{Sel}_{\underline{I}^{c}}\left(M / F_{\infty}\right)$ are cotorsion $\mathbf{Z}_{p}[[\Gamma]]-$ modules. Furthemore, assume that the $\mu$-invariant of $\operatorname{Sel}_{\underline{I}}\left(M^{*} / F_{\infty}\right)$ is zero. Then the $\lambda$-invariant of $\operatorname{Sel}_{\underline{I}}^{\Sigma_{0}}\left(M^{*} / F_{\infty}\right)$ is equal to $\operatorname{dim}_{\mathbf{F}_{p}} \operatorname{Sel}_{\underline{I}}^{\Sigma_{0}}\left(M^{*}[p] / F_{\infty}\right)$.

Proof By Corollary 3.10, $\operatorname{Sel}_{\underline{I}}^{\Sigma_{0}}\left(M^{*} / F_{\infty}\right)$ is a cotorsion $\mathbf{Z}_{p}[[\Gamma]]$-module and its $\mu$-invariant is zero. Thus, the Pontryagin dual of $\operatorname{Sel}_{\underline{I}}^{\Sigma_{0}}\left(M^{*} / F_{\infty}\right)$ is a finitely generated $\mathbf{Z}_{p}$-module, and, by Proposition 3.11, its $\mathbf{Z}_{p}$-torsion submodule is trivial. Thus, $\operatorname{Sel}_{I}^{\Sigma_{0}}\left(M^{*} / F_{\infty}\right)$ is $\mathbf{Z}_{p^{-}}$ divisible with $\mathbf{Z}_{p}$-corank its $\lambda$-invariant (i.e. $\left.\operatorname{Sel}_{\underline{I}}^{\Sigma_{0}}\left(M^{*} / F_{\infty}\right) \simeq\left(\mathbf{Q}_{p} / \mathbf{Z}_{p}\right)^{\lambda}\right)$. Therefore, by Proposition 3.8, we have

$$
\lambda=\operatorname{dim}_{\mathbf{F}_{p}} \operatorname{Sel}_{\underline{I}}^{\Sigma_{0}}\left(M^{*} / F_{\infty}\right)[p]=\operatorname{dim}_{\mathbf{F}_{p}} \operatorname{Sel}_{\underline{I}}^{\Sigma_{0}}\left(M^{*}[p] / F_{\infty}\right) .
$$


We are now able to prove the main result of this section.

Theorem 3.13 Assume (Cong.) and choose compatible good bases for $T$ and $T^{\prime}$ as in Sect. 3.2. Let $\underline{I} \in \mathcal{I}_{p}$ and $\Sigma_{0}$ a finite set of prime as in Definition 3.6 containing the primes of ramification of $M^{*}$ and $M^{\prime}, *$. Further, assume that $\operatorname{Sel}_{\underline{I}}\left(M^{*} / F_{\infty}\right), \operatorname{Sel}_{\underline{I}^{c}}\left(M / F_{\infty}\right), \operatorname{Sel}_{\underline{I}}\left(M^{\prime, *} / F_{\infty}\right)$ and $\operatorname{Sel}_{\underline{I}}\left(M^{\prime} / F_{\infty}\right)$ are cotorsion $\mathbf{Z}_{p}[[\Gamma]]$-modules. Then the $\mu$-invariant of $\operatorname{Sel}_{\underline{I}}\left(M^{*} / F_{\infty}\right)$ vanishes if and only if that of $\operatorname{Sel}_{\underline{I}}\left(M^{\prime}, * / F_{\infty}\right)$ vanishes. Furthermore, when these $\mu$-invariants do vanish, the $\lambda$-invariants of $\operatorname{Sel}_{\underline{I}}^{\Sigma_{0}}\left(M^{*} / F_{\infty}\right)$ and $\operatorname{Sel}_{\underline{I}}^{\Sigma_{0}}\left(M^{\prime, *} / F_{\infty}\right)$ are equal.

Proof The hypothesis (Cong.) implies that $M^{*}[p] \simeq M^{\prime *}[p]$ as $G_{F}$-representations. Hence, we have

$$
\begin{aligned}
\mathrm{H}^{1}\left(F_{\Sigma} / F_{\infty}, M^{*}[p]\right) & \simeq \mathrm{H}^{1}\left(F_{\Sigma} / F_{\infty}, M^{\prime, *}[p]\right), \\
\mathrm{H}^{1}\left(F_{v, \text { unr }}, M^{*}[p]\right) & \simeq \mathrm{H}^{1}\left(F_{v, \mathrm{unr}}, M^{\prime, *}[p]\right),
\end{aligned}
$$

for $v$ not dividing $p$. By Remark 3.7 and Proposition 3.4, for each $v$ dividing $p$, we have

$$
\mathrm{H}_{I_{v}}^{1}\left(F_{v, \infty}, M^{*}[p]\right) \simeq \mathrm{H}_{I_{v}}^{1}\left(F_{v, \infty}, M^{\prime * *}[p]\right) .
$$

It follows that

$$
\operatorname{Sel}_{\underline{I}}^{\Sigma_{0}}\left(M^{*}[p] / F_{\infty}\right) \simeq \operatorname{Sel}_{\underline{I}}^{\Sigma_{0}}\left(M^{\prime, *}[p] / F_{\infty}\right)
$$

Combined with Proposition 3.8, we have

$\operatorname{Sel}_{\underline{I}}^{\Sigma_{0}}\left(M^{*} / F_{\infty}\right)[p] \simeq \operatorname{Sel}_{\underline{I}}^{\Sigma_{0}}\left(M^{*}[p] / F_{\infty}\right) \simeq \operatorname{Sel}_{\underline{I}}^{\Sigma_{0}}\left(M^{\prime, *}[p] / F_{\infty}\right) \simeq \operatorname{Sel}_{\underline{I}}^{\Sigma_{0}}\left(M^{\prime, *} / F_{\infty}\right)[p]$.

Therefore, the first assertion follows from Corollary 3.10. The second claim follows from Corollary 3.12.

As a direct consequence of Theorem 3.13 and Corollary 3.10, we get:

Corollary 3.14 Assume the same hypotheses as in Theorem 3.13 and also assume that

$$
\mu\left(\operatorname{Sel}_{\underline{I}}\left(M^{*} / F_{\infty}\right)\right)=\mu\left(\operatorname{Sel}_{\underline{I}}\left(M^{\prime, *} / F_{\infty}\right)\right)=0 .
$$

Then

$$
\lambda-\sum_{w \in \Sigma_{0}} \operatorname{corank}_{\mathbf{Z}_{p}} \mathrm{H}^{1}\left(F_{\infty, w}, M^{*}\right)=\lambda^{\prime}-\sum_{w \in \Sigma_{0}} \operatorname{corank}_{\mathbf{z}_{p}} \mathrm{H}^{1}\left(F_{\infty, w}, M^{\prime, *}\right),
$$

where $\lambda$ (respectively $\left.\lambda^{\prime}\right)$ denotes the $\lambda$-invariant of $\operatorname{Sel}_{\underline{I}}\left(M^{*} / F_{\infty}\right)$ (respectively $\operatorname{Sel}_{\underline{I}}\left(M^{\prime, *} /\right.$ $\left.F_{\infty}\right)$ ).

Remark 3.15 In Corollary 3.14, we can compute the $\mathbf{Z}_{p}$-coranks of $\mathrm{H}^{1}\left(F_{\infty, w}, M^{*}\right)$ and $\mathrm{H}^{1}\left(F_{\infty, w}, M^{\prime, *}\right)$ thanks to [11, Proposition 2.4]. Let $v$ be the prime of $F$ under $w$ and $\ell$ be the rational prime (different from $p$ ) under $v$. We denote by $\left(\mathcal{M}_{p}^{*}\right)_{F_{v \text {,unr }}}$ the maximal quotient of $\mathcal{M}_{p}^{*}$ on which the group $\operatorname{Gal}\left(\overline{F_{v}} / F_{v}\right.$,unr $)$ acts trivially and we set $P_{v}(X)=$ $\operatorname{det}\left(1-\operatorname{Frob}_{v} X \mid\left(\mathcal{M}_{p}^{*}\right)_{F_{v} \text {,unr }}\right) \in \mathbf{Z}_{p}[X]$. Then the corank of $\mathrm{H}^{1}\left(F_{\infty, w}, M^{*}\right)$ is equal to the multiplicicy of $\ell^{-\left[F_{v}: \mathbf{Q}_{p}\right]}$ as root of $P_{v}(X)$ in $\mathbf{F}_{p}[X]$. 
Acknowledgements Open access funding provided by Max Planck Society. This article is part of the author's Ph.D. thesis at University Laval. The author would like to thank his supervisor Antonio Lei for his support and patient explanation throughout this project, as well as Kazim Büyükboduk, Daniel Delbourgo and Jeff Hatley for their time and help. The author would also like to thank B.D. Kim. Finally, the author thank the anonymous referee for very useful comments and suggestions on an earlier version of the article, which led to many improvements.

Open Access This article is distributed under the terms of the Creative Commons Attribution 4.0 International License (http://creativecommons.org/licenses/by/4.0/), which permits unrestricted use, distribution, and reproduction in any medium, provided you give appropriate credit to the original author(s) and the source, provide a link to the Creative Commons license, and indicate if changes were made.

\section{References}

1. Berger, L.: Bloch and Kato's exponential map: three explicit formulas. Documenta Mathematica. no. Extra Volume, 99-129, Kazuya Kato's fiftieth birthday (2003)

2. Berger, L.: Limites de représentations cristallines. Compos. Math. 140(6), 1473-1498 (2004)

3. Bloch, S., Kato, K.: $L$-functions and Tamagawa numbers of motives numbers of motives, the Grothendieck Festschrift, vol I, Progress in Mathematics, vol. 86, pp. 333-400. Birkhäuser, Boston (1990)

4. Büyükboduk, K., Lei, A.: Coleman-adapted Rubin-Stark Kolyvagin systems and supersingular Iwasawa theory of CM abelian varieties. Proc. Lond. Math. Soc. Third Ser. 111(6), 1338-1378 (2015)

5. Büyükboduk, K., Lei, A.: Integral Iwasawa theory of Galois representations for non-ordinary primes. Math. Zeitschrift 286(1-2), 361-398 (2017)

6. Florian, E.: Ito Sprung, Iwasawa theory for elliptic curves at supersingular primes: a pair of main conjectures. J. Number Theory 132(7), 1483-1506 (2012)

7. Fontaine, J.-M.: Le corps des périodes $p$-adiques. Astérisque. (223), 59-111, (1994). With an appendix by Pierre Colmez, Périodes $p$-adiques (Bures-sur-Yvette, 1988)

8. Fontaine, J.-M., Perrin-Riou, B.: Autour des conjectures de Bloch et Kato : cohomologie galoisienne et valeurs de fonctions $L$, Motives (Seattle, WA, 1991). In: proceedings of Symposia in Pure Mathematics, vol. 55, American Mathematical Society, Providence, pp. 599-706 (1994)

9. Greenberg, R.: Iwasawa theory for $p$-adic representations, algebraic number theory, advanced studies in pure mathematics, vol. 17, pp. 97-137. Academic press, Boston (1989)

10. Greenberg, R.: Iwasawa theory for elliptic curves, arithmetic theory of elliptic curves (Cetraro, 1997), Lecture notes in mathematics, vol. 1716, pp. 51-144. Springer, Berlin (1999)

11. Greenberg, R., Vatsal, V.: On the Iwasawa invariants of elliptic curves. Invent. Math. 142(1), 17-63 (2000)

12. Iovita, A., Pollack, R.: Iwasawa theory of elliptic curves at supersingular primes over $\mathbf{Z}_{p}$-extensions of number fields. J. die Reine und Angewandte Mathematik, (Crelle's J.) 598, 71-103 (2006)

13. Kato, K.: Lectures on the approach to Iwasawa theory for Hasse-Weil $L$-functions via $\mathbf{B}_{\mathrm{dR}}$. Part I., arithmetic algebraic geometry (Trento, 1991), lecture notes in mathematics, vol. 1553, pp. 50-163. Springer, Berlin (1993)

14. Kato, K.: p-adic Hodge theory and values of zeta functions of modular forms. Astérisque. no. 295, ix, 117-290, (2004). Cohomologies $p$-adiques et applications arithmétiques. III

15. Kim, B.D.: The Iwasawa invariants of the plus/minus Selmer groups. Asian J. Math. 13(2), 181-190 (2009)

16. Kim, B.D.: The plus/minus Selmer groups for supersingular primes. J. Aust. Math. Soc. 95(2), 189-200 (2013)

17. Kim, B.D.: Signed-Selmer groups over the $\mathbf{Z}_{p}^{2}$-extension of an imaginary quadratic field. Can. J. Math. 66(4), 826-843 (2014)

18. Kitajima, T., Otsuki, R.: On the plus and the minus Selmer groups for elliptic curves at supersingular primes. Tokyo J. Math. 41(1), 273-303 (2018)

19. Kobayashi, S.-I.: Iwasawa theory for elliptic curves at supersingular primes. Invent. Math. 152, 1-36 (2003)

20. Lei, A., Loeffler, D., Sarah Livia, Z.: Wach modules and Iwasawa theory for modular forms, Asian. J. Math. 14(4), 475-528 (2010)

21. Lei, A., Ponsinet, G.: Functional equations for multi-signed Selmer groups. Ann. Math. du Qué. 41(1), 155-167 (2017)

22. Mazur, B.: Rational points of abelian varieties with values in towers of number fields. Invent. Math. 18, 183-266 (1972) 
23. Mazur, B., Rubin, K.: Kolyvagins systems. Mem. Am. Math. Soc. 168(799), viii+96 (2004)

24. Perrin-Riou, B.: Théorie d'Iwasawa et hauteurs $p$-adiques. Invent. Math. 109, 137-185 (1992)

25. Perrin-Riou, B.: Théorie d'Iwasawa des représentations $p$-adiques sur un corps local. Invent. Math. 115(1), 81-161 (1994). (With an appendix by Jean-Marc Fontaine)

26. Perrin-Riou, B.: $p$-adic $L$-functions and $p$-adic representations, SMF/AMS texts and monographs, vol. 3 , American Mathematical Society, Providence, RI; Société Mathématique de France, Paris, 2000, Translate from the 1995 French original by Leila Schneps and revised by the author

27. Perrin-Riou, B.: Représentations $p$-adiques et normes universelles. I. le cas cristallin. J. Am. Math. Soc 13, 533-551 (2000)

Publisher's Note Springer Nature remains neutral with regard to jurisdictional claims in published maps and institutional affiliations. 